\title{
Gestione del Covid-19 in età pediatrica: documento di consenso
}

\begin{abstract}
SUSANNA ESPOSITO', FEDERICO MARCHETTI2, MARCELLO LANARI ${ }^{3}$, FABIO CARAMELLI ${ }^{4}$, ALESSANDRO DE FANTI ${ }^{5}$, GIANLUCA VERGINE ${ }^{\circ}$, LORENZO IUGHETTI', MARTINA FORNARO ${ }^{8}$, AGNESE SUPPIE ${ }^{\circ}$, STEFANO ZONA ${ }^{10}$, ANDREA PESSION ${ }^{\prime \prime}$, GIACOMO BIASUCCI'2 PER IL GRUPPO DI LAVORO SU COVID-19 IN PEDIATRIA DELLA REGIONE EMILIA-ROMAGNA (RE-CO-PED)*
\end{abstract}

${ }^{1} \mathrm{C}$ linica Pediatrica, Azienda 0 spedaliera-Universitaria, Università di Parma; ${ }^{2} \mathrm{UOC}$ di Pediatria e N eonatologia, 0 spedale di Ravenna, AUSL della Romagna; ${ }^{3} U 0$ Pediatria d'Urgenza, IRCCS Azienda O spedaliero-Universitaria, Policlinico di S. 0 rsola, Bologna; ${ }^{4}$ UOC di Anestesia e Rianimazione Generale e Pediatrica, IRC C S Azienda O spedaliero-Universitaria, Policlinico di S. O rsola, Bologna; ${ }^{5}$ O C di Pediatria, O spedale Santa M aria N uova, AUSL-IRC C S di Reggio Emilia; ${ }^{6}$ nità di Pedia tria, 0 spedale di Rimini, AUSL della Romagna; 'UOC di Pediatria, D.A.I. Materno-infantile, Azienda O spedaliero-Universitaria, Università di Modena e Reggio Emilia; ${ }^{8}$ UOC di Pediatria, 0 spedale di Forlì, AUSL della Romagna; ${ }^{9} \mathrm{C}$ linica Pediatrica, Azienda 0 spedaliero-Universitaria, Università di Ferrara; ${ }^{10}$ Dipartimento di Cure Primarie, AUSL Modena; ${ }^{11} \mathrm{C}$ linica Pediatrica, IRC C S 0 spedale Maggiore Policlinico Sant'O rsola, Università di Bologna; ${ }^{12} \mathrm{UOC}$ di Pediatria e N eonatologia, 0 spedale di Piacenza

L'obiettivo di questo dettagliato documento di indirizzo è quello di sintetizzare in modo pratico i dati accumulati e l'esperienza clinica di questi mesi, evidenziando le priorità scientifiche e assistenziali su Covid-19 nel bambino e nell'adolescente. Non esistono certezze assolute, ma bisogna ragionare su quello che in questo momento è noto, al meglio delle evidenze disponibili.

$\mathrm{D}$ a dicembre 2019 la malattia da Coronavirus 2019 (Covid-19) causata dal virus SARS-CoV-2 si è rapidamente diffusa, diventando la prima pandemia del $21^{\circ}$ secolo per numero di morti (oltre 2.000.000 in tutto il mondo al 2.02.21) $)^{1,2}$.

I bambini sembrano essere meno colpiti rispetto agli adulti, con un quadro clinico meno grave e un tasso di mortalità significativamente più basso e inferiore a $1 \%^{3-6}$. Tuttavia, in età pediatrica possono verificarsi gravi, seppur rare, complicanze, come la sindrome infiammatoria multisistemica temporalmente correlata a Covid-19 (MISC) ${ }^{6}$. Molti aspetti dell'infezione da SARS-CoV-2 anche nei bambini e negli adolescenti rimangono poco chiari e il trattamento ottimale per $i$ casi più gravi non è stato definito. L'obiettivo del Gruppo di Lavoro su Covid-19 in Pediatria della Regione Emilia-Romagna (RE-CO-PED) è stato quello di sintetizzare i risultati degli studi disponibili e condividere l'esperienza clinica maturata per evidenziare priorità assistenziali e di ricerca su Covid-19 in età pediatrica e adolescenziale.

\section{MANAGEMENT OF COVID-19 IN THE PAEDIATRIC AGE: CONSENSUS DOCUMENT \\ (Medico e Bambino 2021 ;40:85-)}

\section{Key words}

Covid-19, Children, Prevention, Diagnosis, Treatment, Consensus document

\section{Summary}

Coronavirus disease 2019 (Covid-19) caused by SARS-CoV-2 has rapidly spread, becoming the first pandemic of the 21 st century by death toll. Children appear to be less affected than adults, with a milder clinical presentation and a significantly lower mortality rate. However, serious complica-tions can occur in childhood, such as Covid-19 temporally related multisystem inflammatory syn-drome (MIS-C). Some aspects of SARS-CoV-2 infection in children and adolescents remain un-clear and the optimal treatment has not been defined. The Working Group on Covid-19 in Paediatrics of the Emilia-Romagna Region (RE-CO-PED) has produced a consensus document with practical recommendations based on a systematic review of the literature and on the clinical experi-ence of the expert group. Evidence is reported regarding prevention measures, diagnostic tools as well as home and hospital therapeutic management of complicated cases (MIS-C). The educational and psychological effects of the pandemic in the paediatric and adolescent age are reported, with the need to define coordinated interventions (between paediatricians, neurospychiatrists, psycholo-gists and educational services) for the prevention and treatment of documented emotional, relational and educational consequences caused by the lockdown, school closures and social distances.

\section{METODI}

È stata eseguita una ricerca narrativa della letteratura utilizzando le seguenti parole chiave: Covid-19, SARSCoV-2, children, paediatric, prevention, diagnosis, MIS-C, treatment nei motori di ricerca biomedica (MEDLI$N E$ ), nei siti di società scientifiche produttrici di linee guida e nella letteratura grigia. E stato creato un Comitato di scrittura composto dai Direttori delle Unità di Pediatria delle province della Regione Emilia-Romagna, in- 
tegrato da altri esperti delle stesse Unità che hanno prodotto una prima bozza di documento. Il lavoro è stato successivamente rivisto e modificato con esperti della Regione Emilia-Romagna, pediatri di famiglia, di comunità e ulteriormente condiviso con il gruppo esteso. Per la necessità di fornire indicazioni tempestive non è stata utilizzata la metodologia GRADE e non vengono fornite raccomandazioni, ma solo una sintesi di indicazioni di buone prassi.

\section{QUALI SONO LE MISURE PREVENTIVE DA UTILIZZARE?}

Le evidenze suggeriscono che il SARS-CoV-2 si diffonde da persona a persona in modo diretto e/o indiretto (attraverso oggetti o superfici contaminate) per contatto stretto con persone infette sintomatiche $o$ asintomatiche attraverso le secrezioni della bocca e del naso (saliva, secrezioni respiratorie o goccioline droplet); la trasmissione per via oro-fecale è invece incerta ${ }^{7,8}$. Valutazioni europee riportano che solo $1-5 \%$ dei casi di Covid-19 riguardano l'età pediatrica ${ }^{9}$ anche se stime mondiali arrivano fino al $13 \%^{10,11}$. Nei bambini e negli adolescenti la malattia si ma- nifesta in maniera meno grave rispetto agli adulti con $96 \%$ dei casi asintomatici o con sintomi lievi-moderati e una letalità nella fascia di età $0-15$ anni pari allo $0,08 \%{ }^{11}$. I dati raccolti nel database del Sistema di Sorveglianza Integrata Covid-19 12 indicano che la popolazione scolastica italiana (età 3-18 anni) rappresenta il 15\% della popolazione nazionale e l'11\% del totale degli infetti da SARS-CoV-2 (dati aggiornati al 27.12.20). La maggior parte dei casi in età scolare $(40 \%)$ si è verificata negli adolescenti (14-18 anni), seguiti dai bambini delle scuole primarie di 6-10 anni $(27 \%)$, dai ragazzi delle scuole medie di 11-13 anni (23\%) e dai bambini delle scuole per l'infanzia di 3-5 anni (10\%). La scuola, sulla base dei dati raccolti dall'ISS, non rientra fra i primi contesti di trasmissione in Italia, che sono nell'ordine quello familiare/ domiciliare, sanitario assistenziale e lavorativo ${ }^{12}$. Rimane, tuttavia, ancora da chiarire con precisione il ruolo di bambini e adolescenti nella diffusione dell'infezione $\mathrm{e}^{13-15}$. I bambini più piccoli (in età prescolare e della scuola primaria) sembrano trasmettere il SARS-CoV-2 meno spesso rispetto agli adolescenti. La trasmissione da parte degli adolescenti può verificarsi con la stessa frequenza degli adulti in contesti familiari e comunitari ${ }^{13}$. Poco si sa in relazione alla diffusione del contagio delle nuove varianti di SARS-CoV- $2^{13}$.

In corso di precedenti pandemia la mancanza di contatti sociali e la perdita delle routine scolastiche sono risultate associate a sequele psicologiche anche sul lungo termine ${ }^{16}$. In relazione alla attuale pandemia gli studi hanno rilevato elevati livelli di ansia, depressione e disturbo post-traumatico da stress nei bambini sottoposti a quarantena ${ }^{17,18}$. Per tali motivi tra i diversi interventi di isolamento sociale attuati come misure preventive per contenere l'epidemia ${ }^{19}$ la chiusura dei Servizi educativi e delle scuole è quella più discussa e ritenuta meno appropriata $^{20}$, in considerazione della ricaduta sociale, comportamentale e psicologica $^{21}$. Gli studi disponibili non depongono per un impatto rilevante dell'apertura delle scuole sulla diffusione dell'epidemia, laddove vengano osservate le misure di prevenzione (uso delle mascherine, distanza fisica, frequente ricambio di aria, igiene delle mani) ${ }^{11,22}$

Le misure preventive raccomandate e da adottare anche in età pediatrica

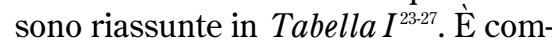
pito del genitore e di ogni servizio educativo quello di sensibilizzare il bambi-

\begin{tabular}{|c|c|c|}
\hline \multicolumn{3}{|c|}{$\begin{array}{l}\text { MISURE PREVENTIVE ATTUALMENTE RACCOMANDATE IN ETÀ PEDIATRICA } \\
\text { IN RELAZIONE ALLE MODALITÁ DI TRASMISSIONE VIRALE }\end{array}$} \\
\hline MODALITÀ DI TRASMISSIONE VIRALE & PREVENZIONE & LIVELLO DI INTERVENTO \\
\hline Interumana (per contatti stretti) & $\begin{array}{l}\text { - Distanziamento sociale di vario grado sino alla chiusura } \\
\text { generalizzata }\end{array}$ & Blocco delle vie di trasmissione del virus \\
\hline Diretta (secrezioni respiratorie) & $\begin{array}{l}\text { - Distanziamento fisico (distanza interpersonale di almeno } \\
\text { un metro anche durante l'attività ludico-sportiva) } \\
\text { - Mascherina facciale (copertura naso, bocca e mento) in } \\
\text { ambienti affollati o poco ventilati e ogni volta che non sia } \\
\text { possibile mantenere il distanziamento, anche di tessuto ma } \\
\text { di adeguate dimensioni. L'O M S ne raccomanda } \\
\text { l'utilizzo a partire dai } 5 \text { anni di età, il CDC già dai } 2 \text { anni } \\
\text { in assenza di disabilità ritenendolo efficace e sicuro }\end{array}$ & \\
\hline $\begin{array}{l}\text { Indiretta (contaminazione di } \\
\text { oggetti superfici, aerosolizzazione) }\end{array}$ & $\begin{array}{l}\text { - Lavaggio corretto e regolare delle mani con acqua e } \\
\text { sapone o con un prodotto a base alcolica } \\
\text { - Pulizia frequente delle superfici } \\
\text { - Aerazione frequente dei locali }\end{array}$ & \\
\hline Infetti (sintomatici e asintomatici) & $\begin{array}{l}\text { - Isolamento } \\
\text { - Tracciamento dei contatti stretti (da } 48 \text { ore antecedenti la } \\
\text { comparsa dei sintomi) }\end{array}$ & Controllo della fonte di infezione \\
\hline Contatti stretti di infetti & - Q uarantena. Sorveglianza attiva & Protezione della popolazione sensibile \\
\hline Tutti i soggetti suscettibili & - Vaccinazione & \\
\hline
\end{tabular}

Tabella I 
no e l'adolescente al rispetto consapevole delle misure di prevenzione in un'ottica di protezione propria e altrui e contemporaneamente stimolare uno stile di vita sano volto a mitigare i disturbi d'ansia, del sonno e i disordini alimentari secondari all'isolamento.

In breve. I bambini si contagiano e trasmettono il SARS-CoV-2 probabilmente con minore frequenza di adolescenti e adulti. In età pediatrica la Covid-19 è comunque meno grave. Valgono per i bambini le stesse misure preventive indicate per la popolazione generale. La prolungata chiusura delle scuole non ha, sulla base dei dati disponibili, un effetto rilevante sulla propagazione della pandemia, mentre si associa a maggiore stress e disagio per bambini e adolescenti.

\section{QUANDO VA ESEGUITO IL TAMPONE PER LA RICERCA DI SARS-COV-2?}

L'indicazione a eseguire un test diagnostico per SARS-CoV-2 dipende da vari fattori che possono modificarsi nel tempo (es. diffusione del virus, tipologia delle misure restrittive, disponibilità di test diagnostici). La presentazione clinica dell'infezione da SARS-CoV-2 in età pediatrica è eterogenea e sostanzialmente sovrapponibile alla maggior parte delle comuni infezioni virali dell'età pediatrica. I sintomi principali sono rappresentati da febbre (nel 59\% dei casi), tosse $(56 \%)$, diarrea $(21 \%)$, congestione nasale e rinorrea (20\%), faringite (18\%), astenia e mialgia (19\%), dispnea $(12 \%)$, nausea e vomito (5\%), cefalea $(4 \%)^{11,28,29}$. L'occorrenza di anosmia, iposmia, ageusia e disgeusia in età pediatrica è inferiore rispetto all'età adulta (1\% nei bambini <10 anni, $10 \%$ dai 10 ai 18 anni) ${ }^{11}$. Più frequentemente, invece, sono state rilevate lesioni cutanee altamente polimorfiche come livedo reticularis, lesioni purpuriche, lesioni morbilliformi, lesioni eritema multiforme-like, cianosi delle estremità e lesioni vescicolari ${ }^{30}$, la cui associazione causale con SARS-CoV-2 rimane comunque incerta ${ }^{11}$. I sintomi gastrointestinali possono non essere associati a sintomi respiratori. Rara la presentazione con quadro grave caratterizzato da MIS- $\mathrm{C}^{31}$.

La letteratura è concorde nell'eseguire l'indagine molecolare nel caso di bambino sintomatico o di contatto stretto $^{29}$. Secondo le indicazioni nazionali si definisce contatto stretto un convivente o persona che sia venuto in contatto con le secrezioni o che abbia avuto un contatto fisico o sia stato faccia a faccia con un soggetto positivo probabile o comprovato, in assenza di dispositivi di protezione, per un tempo superiore a 15 minuti, a una distanza inferiore a 2 metri $^{32}$. Viene, inoltre, raccomandato di eseguire l'esame in tutti i pazienti che necessitano di ricovero in ambiente ospedaliero (screening allargato), anche per motivi non legati allinfezione da SARS-CoV-2 (es. iter diagnostici, procedure, interventi chirurgici) data l'elevata prevalenza e la possibile contagiosità di bambini positivi asintomatici ${ }^{33,34}$. Nel caso di minori che necessitano di accompagnamento lo screening riguarda anche l'accompagnatore adulto. Tale precauzione è raccomandata per evitare focolai intraospedalieri, sebbene dai dati di letteratura si riscontri una positività molto bassa ai test di screening $(<1 \%)^{35}$ ed è ragionevole pensare che tale strategia possa cambiare al mutare del contesto epidemiologico. E inoltre richiesta l'esecuzione di un tampone nelle 48 ore precedenti il rientro in Italia dopo soggiorno in Paesi considerati a rischio ${ }^{36}$.

Non è indicato eseguire il tampone nasofaringeo in caso di bambino con sintomatologia diversa da quella descritta oppure in presenza di altro focus infettivo noto, che non necessiti di ricovero ospedaliero.

In pratica, in un contesto di assistenza territoriale o di Pronto Soccorso il sospetto di infezione da SARSCoV-2 viene posto dal pediatra sulla base di un insieme di valutazioni che devono tenere conto: a) del tipo di sintomatologia clinica; b) della ascrivibilità o meno dei sintomi ad altre potenziali cause (es. febbre senza altra localizzazione, concomitante otite, faringotonsillite, altro). In questi casi è utile iniziare una terapia specifica (quando indicata) raccomandando l'isolamento ed osservando l'evoluzione clinica fino alla guarigione; c) del contesto epidemiologico familiare e sociale (anamnesi positiva per familiare sintomatico; profilo di rischio dei familiari conviventi per età, patologie $\mathrm{e}$ professione; frequenza comunitaria).

In breve. L'esecuzione del tampone per la ricerca di SARS-CoV-2 nella popolazione pediatrica è raccomandata nei soggetti che presentano manifestazioni cliniche sospette per infezione da SARSCoV-2, tenendo conto anche del contesto epidemiologico familiare e sociale. E indicata nei bambini asintomatici contatti stretti di pazienti positivi probabili o confermati; è indicata nei pazienti che richiedono il ricovero ospedaliero e in caso di rientro in Italia da uno dei Paesi definiti a rischio. Non è indicata l'esecuzione del tampone in caso di bambino con sintomatologia che presenti un focus infettivo noto e che non necessiti di ricovero ospedaliero.

\section{QUALI SONO LE MODALITÀ DI RACCOLTA DEI CAMPIONI CON SENSIBILITÀ E SPECIFICITÀ OTTIMALE PER LA DIAGNOSI?}

Dalla segnalazione del primo caso pediatrico di Covid-19, il test in biologia molecolare (RT-PCR) su campioni nasofaringei (NP) e orofaringei (OP) è stato considerato il gold standard per la diagnosi di infezione da SARS-CoV2 in età pediatrica come per la popolazione generale ${ }^{2-4}$. Il test processato in RT-PCR permette di identificare e amplificare sequenze di RNA virale: SARS-CoV-2 può essere ritrovato nel campione biologico a partire da unotre giorni prima dell'insorgenza dei sintomi fino alla seconda settimana dopo l'inizio della sintomatologia ${ }^{22}$. Il rilevamento del virus dipende da numerosi fattori quali: quantità virale nel campione, procedure e tempistica di raccolta, conservazione e processazione del campione ${ }^{36}$. Alcuni studi condotti sulla popolazione adulta hanno dimostrato che SARS-CoV-2 viene rilevato più frequentemente nei campioni prelevati nelle basse vie respiratorie, mentre tra quelli delle alte vie respiratorie il prelievo OP è il meno sensibile ${ }^{37}$. Come affermato dagli esperti dei Centers 
for Disease Control and Prevention (CDC) degli Stati Uniti, le strategie di test e il tipo di campione consigliato sono gli stessi per bambini e adulti ${ }^{38}$. Campioni alternativi a quelli raccolti dal tratto respiratorio (ad esempio da tampone rettale) non vengono raccomandati per le scarse evidenze in età pediatrica e in età adulta ${ }^{39}$. Uno studio prospettico che ha confrontato i tamponi nasali e OP in bambini con Covid19 ha mostrato un tasso di positività significativamente più alto e una carica virale media significativamente più alta sui campioni nasali, supportando la raccolta di campioni nasali invece che $\mathrm{OP}^{40}$. Inoltre, l'aspirato nasofaringeo è stato proposto come alternativa al tampone $\mathrm{NP}^{41}$.

Ad oggi, la maggior parte degli studi si concentra su gruppi di pazienti specifici e la dimensione del loro campione è troppo limitata per raccomandare campioni alternativi, come la saliva, per rilevare l'infezione da SARS-CoV-2. In attesa di studi ulteriori e più ampi per determinare se un metodo di raccolta è più affidabile degli altri per diagnosticare Covid-19 nei bambini, le linee guida e gli esperti concordano nel ritenere che i tamponi NP e quelli OP sono campioni ottimali per diagnosticare l'infezione da SARS-CoV-2 in età pediatrica.

In breve. I tamponi nasofaringei e orofaringei sono di norma utilizzati per diagnosticare l'infezione da SARS-CoV-2 in età pediatrica: il nasofaringeo, fra quelli del tratto respiratorio superiore, appare essere quello con maggiore sensibilità. II test molecolare (RT-PCR) è considerato anche per i bambini e adolescenti il gold-standard.

\section{QUALE GESTIONE DEI CASI SOSPETTI O POSITIVI A SARS-COV-2?}

Come riportato nel documento ministeriale ${ }^{42}$, due sono le possibili misure di restrizione:

- isolamentodeicasididocumentata infezione da SARS-CoV-2: si riferisce alla separazione delle persone infette dal resto della comunità per la durata del periodo di contagiosità, in ambiente e condizioni tali da prevenire la trasmissione dell'infezione;
- quarantena: si riferisce alla restrizione dei movimenti di persone sane, ma che potrebbero essere state esposte a un agente infettivo o a una malattia contagiosa, per la durata del periodo di incubazione con l'obiettivo di monitorare l'eventuale comparsa di sintomi e identificare tempestivamente nuovi casi.

La gestione si differenzia come di seguito riportato.

\section{Casi positivi asintomatici}

Le persone asintomatiche risultate positive alla ricerca di SARS-CoV-2 possono rientrare in comunità dopo un periodo di isolamento di almeno 10 giorni dalla comparsa della positività, al termine del quale risulti eseguito un test molecolare con risultato negativo (10 giorni + test).

\section{Casi positivi sintomatici}

Le persone sintomatiche risultate positive alla ricerca di SARS-CoV-2 possono rientrare in comunità dopo un periodo di isolamento di almeno 10 giorni dalla comparsa dei sintomi accompagnato da un test molecolare con riscontro negativo eseguito dopo almeno 3 giorni senza sintomi (10 giorni, di cui almeno 3 giorni senza sintomi non considerando anosmia e ageusia/disgeusia che possono avere prolungata persistenza nel tempo + test).

\section{Casi positivi a lungo termine}

Le persone che continuano a risultare positive al test molecolare per SARS-CoV-2, in assenza di sintomi (fatta eccezione per ageusia/disgeusia e anosmia che possono perdurare per diverso tempo dopo la guarigione) da almeno una settimana, potranno interrompere l'isolamento dopo 21 giorni dalla comparsa dei sintomi. Questo criterio potrà essere modulato dalle $\mathrm{Au}$ torità sanitarie d'intesa con esperti clinici e microbiologi/virologi, tenendo conto dello stato immunitario delle persone interessate (nei pazienti immunodepressi il periodo di contagiosità può essere prolungato). Per l'Emilia-Romagna è stato indicato di valutare caso per caso quali test per SARS-CoV2 effettuare nei soggetti con immuno- depressione grave (come ad esempio pazienti trapiantati in terapia immunosoppressiva, pazienti oncologici in terapia chemioterapica, pazienti HIV < $200 \mathrm{CD} 4+$, pazienti in trattamento corticosteroideo ad alte dosi assunto nelle settimane precedenti il Covid o in corso ma per motivi diversi dal trattamento di Covid-19) o con malattia Covid-19 grave/critica con prolungata persistenza di attività infiammatoria polmonare.

\section{Contatti stretti asintomatici}

I contatti stretti di casi con infezione da SARS-CoV-2 confermati e identificati dalle Autorità sanitarie, devono osservare:

- un periodo di quarantena di 14 giorni dall'ultima esposizione al caso; oppure

- un periodo di quarantena di 10 giorni dall'ultima esposizione con un test antigenico o molecolare negativo effettuato il decimo giorno.

Si raccomanda di:

- eseguire il test molecolare a fine quarantena a tutte le persone che vivono o entrano in contatto regolarmente con soggetti fragili e/o a rischio di complicanze;

- prevedere accessi al test differenziati per i bambini;

- non prevedere quarantena né l'esecuzione di test diagnostici nei contatti stretti di contatti stretti di caso (ovvero non vi sia stato nessun contatto diretto con il caso confermato), a meno che il contatto stretto del caso non risulti successivamente positivo ad eventuali test diagnostici o nel caso in cui, in base al giudizio delle Autorità sanitarie, si renda opportuno uno screening di comunità.

Si ricorda che è di competenza dei Dipartimenti di Sanità Pubblica l'esecuzione di indagini epidemiologiche, la disposizione dei provvedimenti di isolamento e quarantena, l'organizzazione dei tamponi, la comunicazione delle misure di prevenzione alle famiglie e la segnalazione ai comuni e alla prefettura competente. E però importante che le informazioni siano chiaramente condivise fra tutti i professionisti che assistono il bambino, perché non ci sia 
contraddizione fra quanto raccomandato dai Servizi territoriali e da quelli ospedalieri.

In breve. Le raccomandazioni sui tempi di isolamento in età pediatrica sono assimilabili a quelle dell'adulto. In particolare:

- Il bambino/adolescente positivo asintomatico può terminare l'isolamento dopo almeno 10 giorni dalla comparsa della positività, al termine dei quali venga eseguito un test molecolare con risultato negativo;

- Il bambino/adolescente positivo sintomatico può terminare l'isolamento dopo almeno 10 giorni dalla comparsa dei sintomi e se effettua un test molecolare con riscontro negativo dopo almeno 3 giorni senza sintomi;

- In caso di positività persistente e in assenza di sintomatologia da almeno una settimana, il paziente può terminare l'isolamento dopo 21 giorni dalla comparsa dei sintomi;

- I contatti stretti di casi con infezione da SARS-CoV-2 devono osservare o un periodo di quarantena di 14 giorni dall'ultima esposizione al caso o un periodo di quarantena di 10 giorni dall'ultima esposizione con un test antigenico o molecolare negativo effettuato il decimo giorno;

- Il bambino/adolescente di ritorno da un Paese a rischio oltre a un test negativo effettuato nelle 48 ore precedenti l'ingresso in Italia deve osservare un periodo di quarantena di 14 giorni.

\section{QUAL È L'UTILITÀ DEI TEST MOLECOLARI, ANTIGENICI E SIEROLOGICI?}

Alla luce delle evidenze disponibili, è fondamentale una scelta appropriata dei test diagnostici da utilizzare per SARS-CoV-2. Tra i criteri da considerare nella scelta dei test bisogna valutare non solo la sensibilità e specificità delle singole metodiche, ma anche i tempi di esecuzione, la necessità di un laboratorio disponibile, i $\operatorname{costit}^{43-47}$. Attualmente sono disponibili test molecolari su tampone NP o OP, test antigenici su tampone nasale, NP, OP o campione salivare e test sierologici. Non vi sono indicazioni differenti per la popolazione pediatrica e quella di età adulta. I test molecolari mediante tampone con- sistono in una RT-PCR che individua parti del virus SARS-CoV-2 (frammenti di RNA ${ }^{43,44}$. Questa metodica rappresenta il gold standard per la diagnosi: il risultato può essere ottenuto in 3-5 ore, la sensibilità è del $100 \%$ in condizioni ideali (anche se la performance clinica può essere più variabile), ma sono richiesti personale addestrato, reagenti specifici e macchine costose. I test antigenici rapidi sono, invece, saggi di tipo diretto, qualitativo, che valutano direttamente la presenza del virus nel campione biologico contenendo come substrato un anticorpo specifico in grado di legarsi con antigeni virali di SARS-CoV-2. Sono disponibili come test Point-of-Care (POC), con i risultati visibili ad occhio nudo (la lettura deve essere effettuata in un intervallo di tempo molto definito e preciso) oppure possono essere dotati di uno strumento di lettura automatizzato che fornisce un risultato scritto ${ }^{45}$ ${ }^{47}$; sono disponibili anche come test da eseguire in laboratorio. Sono più veloci ed economici dei test gold standard, ma meno sensibili, in particolare quando si tratta di test di prima e seconda generazione. In particolare, la sensibilità è del 70-86\% e la specificità del 95$97 \%$. I test antigenici rapidi richiedono un campione contenente migliaia di particelle virali per microlitro per dare un risultato positivo. Il test può risultare negativo se la concentrazione degli antigeni è inferiore al limite di rilevamento (es. test eseguito troppo precocemente rispetto all'esposizione o troppo tardivamente rispetto all'insorgenza dei sintomi). Quindi, questi test risultano utili soprattutto per le indagini di screening, quali ad esempio quelle all'interno delle scuole ${ }^{45,46,48}$, dove la bassa probabilità pre-test (bassa prevalenza) determina un valore predittivo negativo elevato. Di converso, il valore predittivo positivo in condizioni di bassa probabilità pre-test è basso e si associa a un elevato tasso di falsi positivi. $\mathrm{Ne}$ consegue che un test positivo antigenico effettuato in ambito di screening dovrebbe essere confermato con un secondo tampone molecolare, rischiando di aumentare il disagio soprattutto nei bambini più piccoli ${ }^{47}$.

I test sierologici forniscono, invece, informazioni di tipo indiretto, cioè rilevano la presenza di anticorpi specifici contro le proteine più immunogeniche di SARS-CoV-2, ossia la proteina spike $(\mathrm{S})$ e/o la nucleoproteina $(\mathrm{N})$, e indicano un'infezione pregressa $o$ in atto $^{49}$. La specificità di questi test varia tra il $96,5 \%$ e il $100 \%$ per i test automatizzati e tra il $94,7 \%$ e il $96,5 \%$ per i test immunoenzimatici rapidi, mentre la sensibilità complessiva varia tra il

\section{UTILTÀ E LIMITI DEI TEST ANTIGENICI E SIEROLOGICI IN ETÀ PEDIATRICA}

\begin{tabular}{|c|c|}
\hline Utilità dei test sierologici & Utilità dei test antigenici \\
\hline $\begin{array}{l}\text { 1. Valutazione di pazienti con un elevato } \\
\text { sospetto clinico per Covid-19 quando il test } \\
\text { molecolare risulta negativo e sono } \\
\text { trascorse almeno due settimane } \\
\text { dall'insorgenza dei sintomi } \\
\text { 2. Monitoraggio della sindrome } \\
\text { infiammatoria multisistemica nei bambini } \\
\text { 3. Conduzione di studi di sierosorveglianza } \\
\text { 4. Potenziale utilità per tracciare il corso della } \\
\text { pandemia di SARS-CoV-2 nella comunità }\end{array}$ & $\begin{array}{l}\text { 1. Utilizzo come test di screening in a mbienti } \\
\text { ad alto rischio } \\
\text { 2. Utilizzo in contesti in cui è necessario un } \\
\text { tempo di risposta rapido come le scuole } \\
\text { primarie e secondarie di primo grado } \\
\text { 3. Utilizzo in a mbienti con ridotta possibilità } \\
\text { di eseguire test RT-PCR per motivi logistici } \\
\text { o economici, essendo meno costosi e più } \\
\text { facilmente utilizzabili }\end{array}$ \\
\hline Limiti dei test sierologici & Limiti dei test antigenici \\
\hline $\begin{array}{l}\text { 1. Scarsa utilità diagnostica in fase acuta di } \\
\text { infezione } \\
\text { 2. Impossibilità di utilizzo nella } \\
\text { determinazione dello sta to immunologico } \\
\text { a causa di mancanza di dati su efficacia } \\
\text { e durata della risposta anticorpale } \\
\text { anti-SARS-CoV-2 }\end{array}$ & $\begin{array}{l}\text { 1. Sensibilità complessiva inferiore a test } \\
\text { basati su RT-PCR } \\
\text { 2. Scarsa utilità diagnostica dopo } 5 \text { giorni } \\
\text { dall'insorgenza dei sintomi }\end{array}$ \\
\hline
\end{tabular}

Tabella II 
$63,2 \%$ e il $73,1 \%$, con prestazioni ottimali dopo quattro settimane dall'insorgenza dei sintomi ${ }^{50}$. Sono utili soprattutto per rilevare l'esposizione al virus, e dunque utilizzati anche in ambito epidemiologico negli studi di sieroprevalenza, non sono invece utili per la diagnosi dell'infezione acuta, proprio perché la positivizzazione avviene settimane dopo l'esposizione/ insorgenza dei sintomi.

In caso di positività è necessaria la rivalutazione con test molecolare per escludere la potenziale contagiosità residua. L'utilità e i limiti dei test antigenici e sierologici in età pediatrica sono riassunti in Tabella II.

In breve. La RT-PCR su tampone rappresenta il gold standard per la diagnosi di SARS-CoV-2. I test antigenici rapidi sono più veloci ed economici dei molecolari, ma meno sensibili e risultano utili soprattutto per le indagini di screening in ambito scolastico. I test sierologici forniscono, invece, informazioni di tipo indiretto, sono utili in ambito epidemiologico negli studi di sieroprevalenza.

\section{QUANDO VA RICOVERATO IL PAZIENTE PEDIATRICO SARS-COV-2 POSITIVO?}

La classificazione dell'Organizzazione Mondiale della Sanità (OMS), ripresa dalla Canadian Paediatric Society ${ }^{51}$, distingue i quadri clinici in base alla gravità:

1. asintomatico-lieve, in caso di presenza di febbre e/o astenia e/o sintomi compatibili con infezione delle alte vie aeree senza necessità di ossigeno e in assenza di evidenza strumentale di polmonite;

2. moderato, in presenza di dispnea e tachipnea senza necessità di ossigeno, sintomi generali (respiratori, gastrointestinali, ageusia, anosmia) ed evidenza strumentale di polmonite;

3. grave, se distress respiratorio grave con desaturazione $\left(\mathrm{SpO}_{2}<92 \%\right.$ in aria ambiente) o tachipnea e/o sintomi sistemici di tipo cardiologico, neurologico, gastrointestinale e coagulativo;

4. critico, se sindrome da distress re- spiratorio acuto (ARDS), insufficienza multiorgano (MOF), shock settico o coma.

La maggior parte dei pazienti pediatrici SARS-CoV-2 positivi presenta quadri clinici asintomatici/lievi. Nonostante la scarsa concordanza delle eviden$\mathrm{ze}^{5,52,53}$, si possono individuare tre fattori che influenzano la decisione di ricoverare un bambino positivo: gravità della malattia, comorbidità e compliance familiare. Uno studio recente svolto in Emilia-Romagna pone l'attenzione sull'importanza di una adeguata gestione territoriale per la riduzione del tasso di ricovero di questi bambini ${ }^{54}$.

Vi è consenso unanime sulla indicazione al ricovero nelle forme da moderate a critiche e nel lattante febbrile al di sotto dei 3 mesi. Nelle forme gravi va sospettata ed esclusa una immunodeficienza primitiva, sulla base di recenti dati che dimostrano mutazioni del toll-like receptor 3 e dell'interferone di tipo 1 in pazienti con grave polmonite da SARS-CoV- $2^{55}$.

I bambini con sintomi lievi possono essere gestiti a domicilio con sorveglianza telefonica, eventuale visita da parte del pediatra curante e/o delle Unità Speciali di Continuità Assistenziale (USCA) pediatriche e istruendo i caregivers sui segni clinici di deterioramento (quali distress respiratorio, dolore toracico, cianosi, alterazione stato coscienza, oliguria). Qualora la compliance familiare risulti non adeguata, è indicato il ricovero.

I bambini con patologie croniche, in particolare malattie polmonari croniche, cardiache, neuromuscolari, oncologiche e deficit immunitari sono a maggior rischio di sviluppare una malattia grave, anche se le casistiche riportate sono limitate e il rischio rimane comunque basso ${ }^{5,53,54}$. In questi casi viene consigliato il ricovero. Nelle forme asintomatiche-lievi è ragionevole una gestione domiciliare con sorveglianza del curante.

In breve. Il ricovero è raccomandato in caso di malattia da moderata a grave, nel lattante febbrile di età inferiore ai 3 mesi e in caso di scarsa compliance familiare. In caso di malattia cronica gra- ve sottostante (malattie polmonari, cardiache, neuromuscolari, oncologiche, deficit immunitari) viene consigliato il ricovero. Nelle forme asintomatiche-lievi è ragionevole una gestione domiciliare con sorveglianza del curante.

\section{SONO RACCOMANDAZIONI SPECIFICHE PER I PAZIENTI CON MALATIIA CRONICA?}

I bambini con malattie croniche sono una popolazione ad alto rischio da un punto di vista infettivo: i casi osservati fino ad ora, però, non hanno dimostrato una maggiore probabilità di sviluppare una malattia grave da SARS-CoV-2 rispetto agli altri bambi$\mathrm{ni}^{56}$. Come suggerimento di buon senso si raccomanda di rafforzare in questi casi l'adozione di comportamenti preventivi specifici per SARS-CoV-2 come rispetto della distanza fisica interpersonale, uso di mascherine di dimensioni adatte e igiene delle mani oltre a misure di tipo generale quali una corretta alimentazione, un adeguato esercizio fisico e un sonno regolare. In base all'andamento della pandemia può essere necessario ridurre i controlli clinici a quelli strettamente necessari utilizzando risorse di telemedicina, ma garantendo un contatto diretto e tempestivo qualora compaiano sintomi di sospetta Covid19 , soprattutto respiratori o gastrointestinali ${ }^{57}$. Il programma di vaccinazione, compresa la vaccinazione contro l'influenza stagionale per le categorie previste, deve proseguire invariato ${ }^{58}$. Non ci sono indicazioni per modificare, ridurre o sospendere le terapie usuali senza una specifica indicazione clinica.

Di seguito vengono riportate indicazioni specifiche per alcune categorie di pazienti.

Pazienti immunocompromessi (es. malattia infiammatoria intestinale, malattia epatica cronica, malattia renale cronica, trapianto di organo, malattia reumatologica). Il rischio di contrarre una infezione da SARS-CoV-2 o di sviluppare una forma grave di Covid-19 sembra essere basso, probabilmente a causa del- 
l'effetto protettivo dei farmaci immunosoppressori che riducono la risposta infiammatoria ${ }^{59.63}$. Le terapie usuali, compresi i biologici, dovrebbero essere proseguite secondo le linee guida.

In presenza di sintomi respiratori moderati-gravi da Covid-19 è indicato il ricovero in ospedale e l'identificazione precoce di qualsiasi segno di polmonite. Le terapie somministrate in corso di Covid-19 dovrebbero tenere conto delle malattie sottostanti (es. filtrato glomerulare in caso di insufficienza renale cronica) e in particolare il dosaggio delle terapie immunosoppressive va rimodulato in base alla gravità dell'infezione e al tipo di farmaci utilizza$\mathrm{ti}^{63}$. Sulla base dell'esperienza clinica si suggerisce di riprendere la terapia immunosoppressiva, se interrotta, dopo due settimane nei pazienti asintomatici o dopo la completa risoluzione clinica nei pazienti sintomatici.

Pazienti con malattie respiratorie croniche. L'asma bronchiale moderato-grave è un fattore di rischio di morbosità e mortalità da Covid-19 $9^{64}$. Gli inalatori predosati o a polvere secca dovrebbero essere preferiti ai farmaci nebulizzati che aumentano il rischio di diffusione del virus. Le esacerbazioni acute dell'asma devono essere trattate tempestivamente e, se necessario, anche con l'uso di corticosteroidi. Nell'asma grave, i farmaci biologici non devono essere sospesi, tranne che durante l'eventuale fase acuta di Covid19. I pazienti con fibrosi cistica, sebbene a rischio di infezioni respiratorie, non mostrano una maggiore prevalenza di infezione da SARS-CoV-2 rispetto alla popolazione generale ${ }^{65}$. Alcuni fattori potrebbero agire come protettivi: l'abitudine alle misure igieniche e di prevenzione, l'uso di farmaci e l'alterata risposta infiammatoria polmonare.

Pazienti con malattie emato-oncologiche. Non è descritta una maggiore incidenza di infezioni rispetto alla popolazione generale $^{66}$. Pertanto, nel rispetto delle regole di igiene e distanziamento previste dalla normativa, va garantito l'accesso alle strutture sanitarie dei pazienti durante la terapia o per le visite urgenti. $\grave{E}$ ancora controverso se la chemioterapia debba essere ritardata nei pazienti asintomatici SARS-CoV-2 positivi.

Pazienti con disturbi neuromuscolari. Analogamente a quanto accade per altre infezioni respiratorie, in particolare l'influenza, in questi pazienti deve essere ricordato il rischio di insufficienza respiratoria durante Covid-1967. Si raccomandano regole di prevenzione, la prosecuzione delle terapie farmacologiche e della fisioterapia respiratoria e attenzione alla comparsa dei sintomi respiratori.

Pazienti con malattie endocrinologiche. I pazienti con diabete di tipo 1 (T1D) sono scarsamente rappresentati tra i soggetti ospedalizzati per Covid- $19^{68,69} \mathrm{e}$ il T1D non sembra predisporre a maggior rischio di infezione da SARS-CoV-2 in età pediatrica e adolescenziale $\mathrm{e}^{70}$. Si raccomanda, comunque, di mantenere un buon controllo glicemico per evitare complicazioni in caso di infezione. A questo scopo la telemedicina ha un ruolo importante, come è stato chiaramente dimostrato ${ }^{71}$, permettendo di mantenere un contatto regolare con i pazienti e di migliorare il controllo metabolico anche durante la quarantena. Il timore del contagio nell'accesso alle strutture sanitarie potrebbe interferire con una diagnosi tempestiva di T1D e provocare un aumento dei casi di grave chetoacidosi diabetica $^{70}$. Quindi, è estremamente importante che i pediatri di famiglia non esitino a indirizzare un bambino con sospetta insorgenza di diabete in un Centro specializzato così da effettuare tempestivamente gli opportuni accertamenti diagnostici e predisporre l'inizio della terapia ${ }^{72}$. Per quel che riguarda l'insufficienza surrenalica (AI), nonostante questi pazienti abbiano un maggiore rischio di infezione e il fabbisogno cronico di glucocorticoidi li possa esporre a un rischio maggiore di malattie virali ${ }^{73}$, non è stato segnalato un maggior rischio di Covid-19. Considerando che l'infezione è una condizione di stress acuto che richiede una dose maggiore di glucocorticoidi e che le crisi surrenaliche durante le infezioni sono le principali cause di morte nei pazienti con $\mathrm{AI}^{74}$, in caso di sintomi da Covid-19 deve essere iniziato un rapido aumento del regime glucocorticoide raddoppiando la dose ${ }^{75}$. Inoltre, è importante consigliare ai pazienti di assumere abbondanti quantità di liquidi contenenti elettroliti. Infine, i pediatri devono tenere presente che se questi pazienti presentano vomito o diarrea, devono essere immediatamente ricoverati in ospedale.

In breve. Nei bambini con cronicità non si evidenzia una maggiore incidenza o gravità di Covid-19. E opportuno comunque rafforzare i messaggi preventivi rivolti a questi bambini e alle loro famiglie. Nelle fasi epidemiche acute si consiglia di ridurre $i$ controlli clinici a quelli strettamente necessari utilizzando risorse di telemedicina, ma garantendo un contatto diretto e tempestivo qualora compaiano sintomi di sospetta Covid19, soprattutto di tipo respiratorio o gastrointestinale. In questi pazienti è raccomandata la vaccinazione contro l'influenza stagionale. Non ci sono indicazioni per modificare, ridurre o sospendere le terapie usuali senza una specifica indicazione clinica.

\section{QUALI SONO I SINTOMI E SEGNI PER FARE LA DIAGNOSI DI MIS-C?}

La MIS-C è una sindrome iperinfiammatoria che segue di 2-6 settimane l'esposizione al SARS-CoV-2. Interessa il bambino e l'adolescente ${ }^{76.78}$, con una incidenza stimata di $2 / 100.000^{31}$ casi. Tale sindrome può avere caratteristiche clinico-laboratoristiche simili ad altre entità quali la malattia di Kawasaki (rispetto alla quale colpisce però una popolazione di età maggiore), la sindrome da shock tossico e la sindrome da attivazione macrofagica ${ }^{76,7880}$. Esistono varie definizioni di MIS-C proposte dall'OMS, dal Royal College of Paediatrics and Child Health (RCPCH) e dal CDC (Tabella III) ${ }^{76,77,81}$ : fra i criteri comuni c'è la presenza di febbre persistente $\left(>38^{\circ} \mathrm{C}\right)$, stato infiammatorio sistemico con elevazione degli indici di flogosi, leucocitosi neutrofila, linfopenia e disfunzione d'organo, unitamente all'evidenza laboratoristica o epidemiologica di infezione da SARS-CoV-2 e l'esclusione di altre cause microbiologiche (Tabelle IV e $V)^{76,77,81}$. 


\section{CLASSIFICAZIONI DELLA MIS-C}

OMS (Organizzazione
Mondiale della Sanità)
Bambino 0 adolescente di età 0-19 anni con febbre $>3$ giorni e 2 delle seguenti caratteristiche:

1. rash 0 congiuntivite non purulenta 0 segni di infiammazione mucocutanea (cavo orale, mani o pie di)

2. ipotensione o shock

3. segni di disfunzione miocardica, pericardite, valvulite 0 anomalie coronariche (incluse alterazioni ecografiche o elevazione della troponina/ N T-proBN P)

4. evidenza di coagulopatia (PT, APTT, elevazione del D-dimero)

5. Problemi gastrointestinali acuti $E$

Elevazione degli indici di infiammazione come la PCR, PCT o la VES

$$
\text { E }
$$

Esclusione di altre cause

microbiologiche di infiammazione inclusa sepsi batterica, sindrome da shock tossico stafilococcica 0 strep to coccica

\section{$E$}

Evidenza di infezione da SARS-CoV-2 (test antigenico 0 sierologia positiva) 0 contatto con paziente con Covid-19 Considerare la MIS-C in pazienti con caratteristiche di malattia di Kawasaki tipica/ atipica o di sindrome da shock tossico

RCPCH (Royal College of Paediatrics
and Child Health)
and Child Health)

Paziente con febbre persistente $\left(>38,5^{\circ} \mathrm{C}\right)$, infiammazione sistemica (neutrofilia, elevazione della PCR e linfopenia) ed evidenza di disfunzione d'organo singola o multipla (shock, disturbi cardiaci, renali, gastrointestinali o neurologici) con caratteristiche aggiuntive*.

Potrebbero essere inclusi pazienti con sintomatologia parzialmente 0 totalmente rispondente ai criteri della malattia di Kawasaki.

Esclusione di ogni altra causa microbiologica inclusa sepsi batterica, sindrome da shock tossico stafilococcica 0 strep tococcica, altre cause infettive di miocardite.

La ricerca di SARS-C oV-2 mediante PCR può risultare positiva o negativa.

\section{* Caratteristiche aggiuntive:}

\section{Clinica}

Molti: richiesta di 02 , ipotensione

Alcuni: dolore addominale, confusione, congiuntivite, tosse, diarrea, cefalea, linfoadenopatia, cambiamenti delle mucose, rigidità nucale, rash, sintomi respiratori, faringite edema dei piedi e delle mani, sincope, vomito

\section{Laboratorio}

Tutti: alterazione del fibrinogeno, D-dimero elevato, ferritina elevata, ipoalbuminemia.

Molti: danno renale acuto, anemia, trombocitopenia, coagulopatia, elevazione IL-10, IL-6, proteinuria, elevazione $C K$ ed $L H$, elevazione trigliceridi, troponina e transaminasi.

\section{Imaging}

Ecocardiografia ed ECG: miocardite, valvulite, effusione pericardica, dilatazione delle arterie coronarie. $R x$ : infiltrati polmonari simmetrici, effusione pleurica Eco addome: colite, ileite, linfoadenopatia, ascite, epatosplenomegalia

TC polmonare con contrasto può evidenziare a neurismi coronarici
CDC (Center for Disease Control and prevention)

Pazienti di età $<21$ anni che presentano febbre, evidenza laboratoristica di infiammazione ed evidenza clinica di severa prostrazione che richiede l'ospedalizzazione e la presenza di due o più organi/ apparati colpiti (cuore, rene, apparato respiratorio, ema to poietico, gastrointestinale, dermatologico o neurologico) Febbre $>38^{\circ} \mathrm{C}$ per $\geq 24$ ore 0 soggettiva riferita da oltre 24 ore. Positività laboratoristica di più di 1 dei seguenti indici: PCR, VES, PCT,

fibrinogeno, D-dimero, ferritina, $L D H$ IL-6; neutrofilia, linfopenia e

ipoalbuminemia.

$\mathrm{N}$ on altre diagnosi plausibili E

Positività laboratoristica per infezione recente 0 in atto per SARS-C OV-2 (positività dei test molecolari,

antigenici o delle indagini sierologiche o contatto con un caso certo di

Covid-1 9 nelle 4 settimane precedenti).

\section{Commento}

I pazienti che soddisfano parzialmente o totalmente i criteri della malattia di Kawasaki dovrebbero essere segnalati se incontrano i criteri della M IS-C. Considera la MIS-C nei casi di morte pediatrica con evidenza di infezione da SARS-CoV-2

\section{Tabella III}

Contrariamente all'adulto, nel paziente pediatrico l'apparato respiratorio risulta poco colpito $(4,5 \%)$ con sintomi aspecifici quali tosse o faringite, mentre predominanti per frequenza (oltre il $70 \%$ dei casi) e gravità sono l'interessamento dell'apparato gastroenterico, con sintomi enteritici fino a quadri di addome acuto, e cardiovascolare con quadri di miopericardite e ipotensione fino allo shock, con necessità di supporto cardiovascolare in ambiente intensivo nel $77 \%$ dei casi ${ }^{82}$. Il coinvolgimento cardiaco può presentarsi con incremento degli enzimi (troponina e pro-BNP rispettivamente nell' $80,9 \%$ e nell' $84,9 \%$ dei casi) e/o come disfunzione ventricolare sinistra (45\%), insufficienza valvolare (mitrale e aortica), pericardite e aritmie ${ }^{79,80,83-89}$. Dilatazioni o aneurismi coronarici nella fase acuta di malattia sono documentati nel 6-24\% dei casi ${ }^{79,80,8487,90-98}$ : sono in genere di grado non severo e regrediscono nella gran parte dei casi $^{99}$. Data la natura sistemica della sindrome, frequente è, inoltre, l'interessamento mucocutaneo con rash, congiuntivite ed edema delle estremità $^{79,10-102}$.

In breve. La MIS-C è una sindrome iperinfiammatoria che segue di 2-6 settimane l'esposizione al SARS-CoV-2. I sintomi e i segni per fare diagnosi sono rappresentati da febbre persistente $\left(>38^{\circ} \mathrm{C}\right)$, stato infiammatorio sistemico con elevazione degli indici di flogosi, leucocitosi neutrofila, linfopenia e disfunzione d'organo, unitamente all'evidenza laboratoristica o epidemiologica di infezione da SARS-CoV-2 ed esclusione di altre cause microbiologiche.

\section{QUANDO Ė INDICATA LA TERAPIA SOLO SINTOMATICA E CON QUALI FARMACI?}

Come precedentemente riportato, l'infezione da SARS-CoV-2 nei bambini decorre nella maggioranza dei casi in maniera asintomatica o con sintomi lievi controllabili, quando necessario, con la sola terapia sintomatica eseguibile a domicilio ${ }^{103}$. Nei pazienti di età inferiore a 18 anni i sintomi di presentazione che possono meritare un trattamento sono più comunemente la tosse, la faringodinia, la febbre (la cui frequenza si riduce all'aumentare dell'età), la rinorrea, il vomito, la diarrea, la cefalea. Più raramente può esserci una difficoltà respiratoria che va valutata in merito alla necessità $o$ meno di ricovero ospedaliero. 


\begin{tabular}{|c|c|}
\hline \multicolumn{2}{|r|}{ SEGNI E SINTOMI DELLA MIS-C } \\
\hline $\begin{array}{l}\text { CUORE } \\
\text { * in caso di dilatazione } \\
\text { delle coronarie si } \\
\text { raccomanda di } \\
\text { utilizzare } \\
\text { i criteri dell'American } \\
\text { Heart Association } \\
\text { per la loro definizione }\end{array}$ & $\begin{array}{l}\text { Ipotensione } \\
\text { Shock (possono essere presenti i segni della capillary leak o dello } \\
\text { shock cardiogeno) } \\
\text { M iocardite (anche solo elevazione degli enzimi cardiaci in } \\
\text { assenza di chiari segni ecocardiografici) } \\
\text { Insufficienza valvolare } \\
\text { Insufficienza cardiaca, alterazione delle coronarie (iper-riflettenza, } \\
\text { aumento delle dimensioni) }\end{array}$ \\
\hline RESPIRATORIO & $\begin{array}{l}\text { Congestione nasale, faringodinia e/ o iperemia faringea } \\
\text { Tosse, dolore toracico } \\
\text { Segni di distress respiratorio, insufficienza respiratoria acuta }\end{array}$ \\
\hline CUTE E MUCOSE & $\begin{array}{l}\text { Rash polimorfo/ eritema perineale } \\
\text { Eritema palmo-plantare/ edema del dorso della mano e del piede } \\
\text { Cheilite/ lingua a fragola } \\
\text { Congiuntivite bulbare non secretiva } \\
\text { Linfoadenopatia }\end{array}$ \\
\hline RENE & Insufficienza renale, oliguria/ a nuria, edemi declivi \\
\hline GASTRO-INTESTINALE & $\begin{array}{l}\text { Dolore addominale severo, diarrea, nausea e/ o vomito } \\
\text { Ittero, epatite, idrope della colecisti }\end{array}$ \\
\hline MUSCOLO-SCHELETRICO & Artralgie, mialgie, artrite \\
\hline SNC & Cefalea, irritabilità, meningismo, confusione mentale, crisi convulsive \\
\hline
\end{tabular}

Tabella IV

Il trattamento sintomatico è lo stesso che viene utilizzato per le comuni infezioni respiratorie e per la gastroenterite. In particolare, per la febbre e la sintomatologia dolorosa è indicato l'uso del paracetamolo ${ }^{104-107}$. In merito all'uso dei FANS (ibuprofene), che è stato oggetto inizialmente di controversie $^{107}$, l'EMA, l'OMS e l'AIFA, sulla base dei risultati di studi osservazionali, non ne controindicano il possibile uti$\operatorname{lizzo}^{103-108}$. I pazienti che già assumono regolarmente i FANS per altre patologie non dovrebbero interromperli. Come con qualsiasi farmaco la prescrizione deve tenere conto dei benefici e dei rischi di eventi avversi noti valutati su base individuale. In particolare, l'uso dei FANS è controindicato in presenza di un presunto o documentato stato di disidratazione per il rischio aumentato di insufficienza renale ${ }^{107}$.

I sintomi respiratori possono beneficiare di terapia inalatoria con cortisonici e/o broncodilatatori: in questi casi, come già affermato, è da preferire il distanziatore al nebulizzatore per ridurre la diffusione di particelle virali nell'aria ${ }^{52,109}$. In caso di diarrea o vomito è necessario assicurare una corretta idratazione con soluzioni reidratanti orali.

Non è indicata la terapia antibiotica se non nei casi che possono avere una complicanza batterica. Recenti studi sui pazienti adulti hanno documentato il largo e improprio utilizzo della terapia antibiotica che potrebbe essere una causa rilevante dell'aumento delle resistenze batteriche ${ }^{110}$. Non è noto in età pediatrica il numero di casi con una coinfezione o una complicanza batterica. Nel paziente adulto risulta essere estremamente basso. Una metanalisi che ha incluso 24 studi per un totale di 3338 pazienti con Covid-19 quasi esclusivamente adulti ${ }^{111}$ ha stimato il rischio di una concomitante infezione batterica e ha documentato che tale coinfezione - all'inizio della sintomatologia - era presente nel 3,5\% dei casi e che una infezione batterica secondaria si è verificata nel $14,3 \%$. La percentuale complessiva di pazienti Covid-19 con infezione batterica è stata del 6,9\%; l'infezione batterica è risultata più comune nei pazienti critici $(8,1 \%)$. È importante sottolineare che in realtà la maggior parte dei pazienti con infezione da SARS-CoV-2 ha ricevuto antibiotici (72\%).

Sulla base di quelle che sono le esperienze nei pazienti adulti non è indicato l'uso dell'azitromicina nell'infezione da SARS-CoV-2, sia nelle fasi precoci dell'infezione che in quelle più gravi ${ }^{112,113}$.
In breve. Nella maggioranza dei casi sintomatici di infezione da SARS-CoV-2 in età pediatrica e adolescenziale, è raccomandata la sola terapia sintomatica con paracetamolo o eventualmente con ibuprofene dopo aver escluso uno stato di disidratazione. In caso di sintomi respiratori che possono beneficiare di terapia inalatoria con broncodilatatori e/o cortisonici, è da preferire il distanziatore al nebulizzatore per ridurre la diffusione di particelle virali nell'aria. In caso di diarrea o vomito, va assicurata una corretta idratazione con soluzioni reidratanti orali. Non è indicata la terapia antibiotica se non in presenza di una verosimile complicanza batterica. Non è indicato l'uso dell'azitromicina.

\section{QUANDO SONO INDICATI I FARMACI IMMUNOMODULANTI E COME IMPIEGARLI?}

Nei bambini con condizioni cliniche moderate-gravi, con polmonite e progressione nel deterioramento della funzionalità respiratoria, sindrome da distress respiratorio acuto (ARDS) o condizioni cliniche che rientrano nella diagnosi della MIS-C, alla terapia di supporto va considerata l'aggiunta di una terapia immunomodulante ${ }^{114-117}$.

In particolare, l'uso dei corticosteroidi è raccomandato per gli adulti con infezione da SARS-CoV-2 e con condizioni cliniche gravi che necessitano di supplementazione di ossigeno ${ }^{118}$. La terapia steroidea in questi pazienti riduce la mortalità e migliora il decorso clinico ${ }^{114}$. ${ }^{117}$. Sui bambini con infezione polmonare che richiede supplementazione di ossigeno gli studi sono ancora in corso e i benefici/rischi sono ancora incerti.

In caso di condizioni cliniche gravi o in caso di rapido peggioramento clinico in merito alla sintomatologia respiratoria $\left(\mathrm{SpO}_{2}<92 \%\right)$, rifacendosi al modello dell'adulto, si possono somministrare glucocorticoidi per una durata di 10 giorni o fino alla dimissione, a seconda di quale sia la durata più breve $\mathrm{e}^{117}$. Non c'è comunque al momento un accordo unanime su quale deve essere la durata ottimale della terapia, che andrà pertanto valutata caso per $\operatorname{caso}^{118}$. Le molecole di steroide che possono essere uti- 


\section{ESAMI DI LABORATORIO/STRUMENTALI IN CORSO DI MIS-C}

\begin{tabular}{|c|c|}
\hline ESAMI EMATICI & $\begin{array}{l}\text { Emocromo con formula: leucocitosi con linfopenia } \\
\text { In caso di leucopenia, piastrinopenia o anemia considerare l'ipotesi di SHLH/ MAS } \\
\text { PCR, VES e PCT: elevate } \\
\text { Ferritina: elevata } \\
\text { In caso di VES bassa con PCR elevata o iperferritinemia considerare l'ipotesi di SHLH/ MAS } \\
\text { Coagulazione: fibrinogeno elevato, D-Dimero elevato } \\
\text { Valutare PT e PTT per eventuali alterazioni in senso pro-trombotico } \\
\text { In caso di ipofibrinogenemia considerare l'ipotesi di sHLH/ M AS } \\
\text { Elettroliti: possibile iponatriemia } \\
\text { Funzionalità epatica: in caso di ipertransaminasemia considerare l'ipotesi di SSHLH/ MAS. } \\
\text { Sono descritti casi di M IS-C con idrope della colecisti, che può manifestarsi con iperbilirubinemia } \\
\text { Funzionalità renale: sono descritti casi di M IS-C con insufficienza renale } \\
\text { Troponine e Pro-BNP: elevati in caso di interessamento cardiaco } \\
\text { Proteine totali, albumina sierica: può essere presente ipoalbuminemia } \\
\text { Trigliceridi: in caso di ipertrigliceridemia considerare SHLH/ M AS } \\
\text { CPK, LDH: possono essere aumentate, evidenziano l'eventuale citolisi } \\
\text { IL 6: elevata } \\
\text { C3, C4: può essere presente ipocomplementemia da consumo } \\
\text { YGT: in associazione agli altri esami di funzionalità epatica (primo livello) può testimoniare l'eventuale epatopatia } \\
\text { Funzionalità pancreatica: sono descritti casi con aumento lipasi e amilasi } \\
\text { Esame urine: può essere presente leucocituria in assenza di elementi tipici per infezione delle vie urinarie, segni di } \\
\text { danno tubulare. } \\
\text { Emogas: acidosi metabolica. Aumento dei lattati } \\
\text { Striscio periferico: è descritta la presenza di schistociti o cellule di Burr, che testimoniano la microangiopatia }\end{array}$ \\
\hline $\begin{array}{l}\text { ESAMI MICROBIOLOGICI } \\
\text { N ella maggior parte dei casi } \\
\text { la diagnosi differenziale } \\
\text { principale si pone con la sepsi }\end{array}$ & $\begin{array}{l}\text { Emocoltura, urinocoltura, coprocoltura } \\
\text { Sierologie } \\
\text { In caso di sierologia positiva, laddove disponibile, utile conferma con PCR } \\
\text { AN F per Influenza A e B, VRS e adenovirus }\end{array}$ \\
\hline ESAMI STRUMENTALI & $\begin{array}{l}\text { ECG + ecocardiogramma: ipocinesie in caso di miocarditi, insufficienza valvolare da miocardite, pericardite, } \\
\text { alterazioni delle coronarie. In caso di paziente con shock può essere utile anche per valutare lo stato di idratazione } \\
\text { Rx torace: polmonite interstiziale. Può essere presente pleurite e aumento dell'ombra cardiaca da versamento } \\
\text { pericardico. } \\
\text { Ecografia addome: presenza di organomegalia, versamento peritoneale, idrope della colecisti } \\
\text { TC torace: in caso sia necessario uno studio approfondito della localizzazione polmonare } \\
\text { RMN cardiaca: può essere utile, dove disponibile, in caso di comprovata miocardite } \\
\text { Colonscopia: in casi selezionati con severo coinvolgimento intestinale }\end{array}$ \\
\hline
\end{tabular}

Tabella V

lizzate sono il desametasone $(0,15-0,4$ $\mathrm{mg} / \mathrm{kg} /$ die per os/iv, dose massima 6 $\mathrm{mg})$, il prednisone $(1-2 \mathrm{mg} / \mathrm{kg} /$ die per os, dose massima $60 \mathrm{mg}$ ) o il metilprednisolone $(1-2 \mathrm{mg} / \mathrm{kg} /$ die iv, dose massima $60 \mathrm{mg})^{52,114,117}$. Nei casi di MISC grave (vedasi paragrafo specifico) può essere previsto l'utilizzo dei boli di metilprednisolone $(10-30 \mathrm{mg} / \mathrm{kg} / \mathrm{die}$, dose massima $1 \mathrm{~g}$ ).

Sulla base di quelli che sono i risultati degli studi clinici controllati sempre nella popolazione adulta, il tocilizumab, anticorpo monoclonale diretto contro $\mathrm{i}$ recettori dell'interleuchina (IL)-6, non è indicato nelle forme moderate-gravi di insufficienza respiratoria ${ }^{19,120}$ e utilizzabile esclusivamente nell'ambito di studi clinici ${ }^{108}$.

Anche l'utilizzo dell'anakinra, inibitore dell'IL-1, per il quale non esistono prove di efficacia da studi randomizzati controllati, ma solo case report e studi osservazionali, può essere considerato come trattamento di seconda linea nei casi di MIS-C (vedi paragrafo specifico) e nei casi con un quadro di ARDS ${ }^{121}$. Anche in questo caso AIFA raccomanda di utilizzare il farmaco nell'ambito di studi clinici ${ }^{108}$.

L'anticorpo monoclonale bamlanivimab, diretto contro la proteina spike di SARS-CoV-2, agirebbe bloccando l'attacco del virus e pertanto il suo utilizzo è limitato alle forme lievi-moderate e alle fasi inziali dell'infezione ${ }^{122}$. Uno studio di fase II randomizzato verso placebo ha dimostrato l'efficacia del farmaco nel ridurre la carica virale, e nel migliorare la sintomatologia clinica riducendo il ricorso alla ospedalizzazione solo quando usato in associazione a etesevimab ${ }^{123}$. A seguito del parere favorevole espresso dalla CTS di AI-
$\mathrm{FA}^{124}$, il Ministero della Salute con proprio decreto ha autorizzato l'importazione e l'utilizzo sia negli adulti che nei pazienti pediatrici a partire dai 12 anni di età nelle fasi precoci della Covid-19 in presenza di sintomi lievi moderati, ma con fattori di rischio per evoluzione verso una maggior gravità della malattia, sia di bamlanivimab che della associazione di anticorpi nota come $R E$ GEN-COV.

In particolare per quanto riguarda la fascia di età tra 12 e 17 anni l'uso può essere considerato in pazienti non ospedalizzati per Covid-19, con sintomi di grado lieve-moderato di recente insorgenza (e comunque da non oltre 10 giorni) e con la presenza di almeno uno dei seguenti fattori di rischio: BMI $\geq 85^{\circ}$ percentile per età e genere; anemia falciforme; malattie cardiache congenite o acquisite; malattia del neuro- 
sviluppo; dipendenza da dispositivo tecnologico (es. soggetti con tracheotomia, gastrostomia ecc.); asma, o altre malattie respiratorie che richiedono medicazioni giornaliere per il loro controllo $^{124}$. Dovranno essere definite le modalità organizzative per l'approvvigionamento e la somministrazione degli anticopri monoclonali.

In merito ad altre terapie immunomodulanti che sono state proposte, nei pazienti adulti le evidenze disponibili non supportano: l'idrossiclorochina, sia per un uso preventivo ${ }^{125,126}$ che terapeutico, da sola o in associazione con l'azitromicina ${ }^{126,127}$; l'interferone ${ }^{128}$ e l'utilizzo del plasma da donatori ${ }^{129}$. Per questi ultimi non è raccomandato l'utilizzo in età pediatrica se non in corso di studi clinici (attualmente in corso) ${ }^{124}$.

In breve. Nei bambini con condizioni cliniche gravi, con polmonite e progressione nel deterioramento della funzionalità respiratoria, sindrome da distress respiratorio acuto (ARDS) o condizioni cliniche che rientrano nella diagnosi della MIS-C, alla terapia di supporto va considerata l'aggiunta di una terapia immunomodulante. In caso di condizioni cliniche gravi o in caso di rapido peggioramento clinico in merito alla sintomatologia respiratoria $\left(\mathrm{SpO}_{2}<92 \%\right)$, si possono somministrare glucocorticoidi a basso dosaggio per una durata di 10 giorni o fino alla dimissione, a seconda di quale sia la durata più breve. L'utilizzo dell'anakinra può essere tenuto in considerazione nel trattamento di seconda linea nei casi di MIS-C e nei casi con un quadro di ARDS pur in assenza di prove di efficacia e preferibilmente nell'ambito di studi clinici. L'utilizzo di anticorpi monoclonali nelle fasi iniziali della malattia può essere considerato nel contesto di studi clinici e nei pazienti con età tra 12-17 anni non ospedalizzati, non in ossigenoterapia, con sintomi di grado lieve-moderato di recente insorgenza, in presenza di determinati fattori di rischio. Non è raccomandato l'uso del tocilizumab e non vi sono evidenze a supporto della terapia con idrossiclorochina sia per un uso preventivo che terapeutico da sola o in associazione con l'azitromicina, non è dimostrata l'efficacia dell'interferone né quella del plasma da donatori.

\section{QUANDO È INDICATA LA TERAPIA ANTICOAGULANTE E CON QUALI FARMACI?}

L'infezione da SARS-CoV-2 può essere associata ad uno stato di ipercoagulabilità e coagulazione intravasale disseminata (CID) come conseguenza della tempesta citochinica che si scatena in caso di evoluzione della malattia verso la forma grave ${ }^{130-132}$. Ci sono sempre più evidenze che suggeriscono come l'insieme di disregolazione immunitaria, endotelite e stato ipercoagulativo possa determinare una trombosi microvascolare nel parenchima polmonare e la conseguente insufficienza respiratoria grave. Alcune linee guida raccomandano, pertanto, l'utilizzo profilattico di eparina a basso peso molecolare nei pazienti adulti ricoverati. Non è stata ancora definita la dose da utilizzare a scopo profilattico, in particolare non è noto se sia più efficace la dose standard o una dose più elevata, per cui la posologia va definita caso per caso. In pazienti con sospetta trombosi/embolia dovranno essere utilizzate dosi terapeutiche, come da indicazione registrata ${ }^{133,134}$.

L'incidenza di eventi tromboembolici in corso di Covid-19 nei bambini non è nota. Da un Registro americano si evince una incidenza del $7 \%(3 / 45)$ tra i pazienti di età compresa tra $13 \mathrm{e}$ 21 anni e dell' $1,3 \%(1 / 75)$ tra i 5 e i 13 anni ${ }^{135}$. Tutti i bambini che hanno presentato eventi tromboembolici in corso di Covid-19 avevano più di un fattore di rischio per trombosi ${ }^{133}$.

Una recente Consensus americana suggerisce di iniziare la profilassi antitrombotica nei bambini ricoverati con malattia grave, che abbiano almeno un fattore di rischio per trombosi o un dosaggio ematico di D-dimero elevato ( $\geq$ 5 volte al valore normale), in assenza di controindicazioni (vedi paragrafo sulla MIS-C). La profilassi è consigliata con l'eparina a basso peso molecolare a bassa dose 2 volte al giorno (target del livello di attività anti-Xa a 4 ore dopo la somministrazione da 0,2 a $0,5 \mathrm{U} / \mathrm{ml}$ ) oppure di eparina non frazionata in infusione continua (target del livello di attività anti-Xa da 0,1 a 0,35 U/ml).

La profilassi antitrombotica è sconsigliata in presenza di tromboci- topenia (piastrine $<20.000-50.000 /$ $\mu l$ ), ipofibrinogemia $<100 \mathrm{mg} / \mathrm{dl}$, recente sanguinamento maggiore e terapia con aspirina ad una dose $>5$ $\mathrm{mg} / \mathrm{kg} /$ die. La profilassi anti-trombotica è indicata dopo la dimissione per un ulteriore periodo che deve essere indicato (es. altri 30 giorni) in caso di D-dimero elevato alla dimissione o persistenza di fattori di rischio per trombosi. La profilassi antitrombotica non è al contrario indicata di routine in tutti i bambini ricoverati per Covid-19.

In breve. La profilassi antitrombotica con eparina è raccomandata nei bambini ricoverati con malattia grave, che abbiano almeno un fattore di rischio per trombosi o un dosaggio ematico di D-dimero elevato ( $\geq 5$ volte al valore normale), in assenza di controindicazioni.

\section{QUANDO È INDICATA LA TERAPIA ANTIVIRALE E CON QUALI FARMACI?}

La terapia antivirale per Covid-19 non è necessaria per la grande maggioranza dei pazienti pediatrici ed è raccomandata per i casi gravi e critici, come definiti in precedenza ${ }^{52,136-138}$. Tra gli antivirali, remdesivir è l'unico farmaco registrato per l'uso nei pazienti a partire dai 12 anni di età e peso di almeno $40 \mathrm{~kg}$ con Covid-19 e polmonite che richiedono ossigenoterapia supplementare (ossigeno a basso o alto flusso o altro tipo di ventilazione non invasiva all'inizio del trattamento). AIFA stabilisce che remdesivir potrà essere considerato esclusivamente in casi selezionati dopo un'accurata valutazione del rapporto benefici/rischi in quanto i dati attualmente disponibili non sono concordanti e complessivamente non dimostrano un chiaro beneficio clinico in termini di mortalità o ricorso alla ventilazione meccanica. L'OMS, infatti, dopo aver condotto uno studio randomizzato ed aver eseguito una analisi delle prove di efficacia su oltre 7000 pazienti, ha formulato una raccomandazione negativa debole sull'uso del farmaco nell'intera popolazione di pazienti Covid-19 indipendentemente dalla gravità. 
Nel nostro Paese ${ }^{139}$ l'utilizzo di remdesivir nei pazienti con meno di 12 anni è attualmente possibile esclusivamente:

- attraverso un uso compassionevole: le richieste per il singolo paziente vanno effettuate attraverso l'apposita sezione del sito di Gilead (https://rdvcu.gilead.com/) e vengono valutate ed autorizzate direttamente dalla Ditta caso per caso;

- nell'ambito di trial clinici approvati (https://www.aifa.gov.it/sperimentazioni-cliniche-covid-19).

Remdesivir si lega alla RNA polimerasi RNA-dipendente virale, inibendo la replicazione virale attraverso l'interruzione prematura della trascrizione dell'RNA e dimostrando attività in vitro contro il SARS-CoV-2 ${ }^{140}$. Secondo quanto previsto da FDA il dosaggio raccomandato è: in bambini di peso $>40 \mathrm{~kg}$, il $1^{\circ}$ giorno $200 \mathrm{mg}$ ev in $30 \mathrm{~min}$, seguiti da $100 \mathrm{mg}$ ev/die per altri 9 giorni; in bambini di peso $<40 \mathrm{~kg}$, il $1^{\circ}$ giorno $5 \mathrm{mg} / \mathrm{kg}$ ev in $30 \mathrm{~min}$, seguito da $2,5 \mathrm{mg} / \mathrm{kg}$ ev (in $30 \mathrm{~min}$ )/die per altri 9 giorni. Al momento, il dosaggio non è stato stabilito per le prime 2 settimane di vita e se il peso è $<2,5 \mathrm{~kg}^{140}$. La terapia può essere proseguita per 10 giorni, anche se una durata di 5 giorni è appropriata per la maggior parte dei pazienti ${ }^{140}$.

La sicurezza e l'efficacia della terapia di associazione di remdesivir con corticosteroidi non è stata a oggi studiata in modo adeguatamente approfondito; tuttavia, ci sono ragioni teoriche per cui la terapia di combinazione può essere utile in alcuni pazienti con Covid-19 grave $^{141}$.

Remdesivir, se pur ben tollerato, può causare sintomi gastrointestinali (es. nausea), reazioni di ipersensibilità, livelli elevati di transaminasi e un aumento del tempo di protrombina. Sono in corso studi tesi a valutare l'efficacia di remdesivir associato a tocilizumab o baricitinib nell'adulto.

L'associazione lopinavir/ritonavir, inibitore della proteasi potenziato utilizzato nella terapia dell'infezione da HIV, a partire dall'età di 14 giorni di vi$\mathrm{ta}^{142}$, è da prevedersi solo nel contesto di studi clinici, vista la mancanza di efficacia riportata in letteratura.
Il ruolo antivirale dell'idrossiclorochina, che sembra agire aumentando il $\mathrm{pH}$ endosomiale richiesto per la fusione virus/cellula ospite e interferendo con la glicosilazione del recettore cellulare di SARS-CoV- $2^{143}$, non è stato documentato né negli adulti né nei bambini con Covid-19. AIFA non raccomanda l'utilizzo di idrossiclorochina nei pazienti adulti ricoverati, inoltre non ritiene utile né opportuno autorizzare nuovi studi clinici in tale setting ${ }^{143}$. Per quanto riguarda l'uso sul territorio nelle fasi iniziali della malattia AIFA non raccomanda l'uso dell'idrossiclorochina ${ }^{103}$; un suo eventuale impiego è da considerare off-label.

In breve. La terapia antivirale per Covid19 con remdesivir non è necessaria per la grande maggioranza dei pazienti pediatrici; per i casi gravi e critici è possibile considerarla pur in assenza di chiari dati di efficacia. Il farmaco è disponibile attraverso un Registro web-based AIFA a partire dai 12 anni di età e $40 \mathrm{~kg}$ di peso, al di sotto di tali età e peso nell'ambito di un programma di uso compassionevole o all'interno di trial clinici approvati. Non è raccomandata la terapia con lopinavir/ritonavir o con idrossiclorochina.

\section{QUALE TERAPIA È RACCOMANDATA NELLA MIS-C?}

Mancando delle linee guida condivise sull'argomento, le raccomandazioni sulla gestione terapeutica si basano sull'esperienza clinica di gestione di pazienti con MIS-C e su informazioni disponibili per altre condizioni pediatriche con caratteristiche simili (modello malattia di Kawasaki, MK). Al momento sono disponibili due Consensus sulla gestione della MIS-C: una elaborate da un gruppo di 98 esperti inglesi ${ }^{144}$ e l'altra dall'American College of Rheumato$\log y(\mathrm{ACR})^{145}$. E disponibile anche il documento a cura del gruppo di studio Reumatologia della SIP (www.sip.it).

In merito alla profilassi tromboembolica è stata pubblicata la Consensus della Pediatric/Neonatal Hemostasis and Trombosis Subcommittee of the ISTHSSC ${ }^{135}$.
L'obiettivo del trattamento della MIS-C è ridurre l'infiammazione sistemica e ripristinare la funzione d'organo. Vengono distinti due modelli fenotipici: uno MK-like, clinicamente simile alla MK, e un quadro senza uno specifico fenotipo ma che rientra nei criteri classificativi della MIS-C.

La strategia terapeutica si basa su tre aspetti: 1) trattamento immunomodulante dell'infiammazione; 2) misure di supporto (trattamento dello shock, terapia antibiotica, gastroprotezione); 3) gestione dello stato pro-coagulativo.

\section{Trattamento immunomodulante}

Consiste nell'utilizzo di immunoglobuline, corticosteroidi o farmaci biologici (Tabella VI). Alla luce della sovrapposizione fenotipica con la MK, la terapia anti-infiammatoria di prima linea prevede la somministrazione di Immunoglobuline iv (IVIG) alla dose di $2 \mathrm{~g} / \mathrm{kg}$. Una seconda dose di IVIG è prevista nei casi non responsivi o parzialmente responsivi alla prima infusione ${ }^{14,146-148}$.

La Consensus britannica ${ }^{144}$ prevede l'uso di corticosteroidi ad alte dosi in aggiunta alle IVIG solo nei pazienti con quadro MK-like ad alto rischio secondo il protocollo per la MK ${ }^{149}$. La Consensus dell'ACR ${ }^{145}$ prevede:

- un approccio graduale nella terapia immunomodulatoria;

- IVIG da considerare come terapia di primo livello;

- utilizzare IVIG ad alto dosaggio (tipicamente $2 \mathrm{~g} / \mathrm{kg}$, dosato in base al peso corporeo ideale).

- funzionalità cardiaca e stato dei fluidi devono essere valutati nei pazienti con MIS-C prima di iniziare il trattamento con IVIG. I pazienti con funzione cardiaca depressa richiedono un monitoraggio attento e l'uso dei diuretici concomitante alla somministrazione di IVIG;

- in alcuni pazienti con disfunzione cardiaca, le IVIG possono essere somministrato in dosi frazionate $(1 \mathrm{~g} / \mathrm{kg}$ al giorno per 2 giorni);

- i glucocorticoidi a basso-moderato dosaggio devono essere somministrati con IVIG come terapia aggiuntiva per il trattamento di MIS-C in pazienti con interessamento di organi ed apparati (ad esempio con coinvol- 
gimento cardiaco, gastrointestinale, neurologico);

- nei pazienti che non rispondono a IVIG e glucocorticoidi a dose bassamoderata, vanno presi in considerazione i glucocorticoidi ad alte dosi; i glucocorticoidi ad alte dosi dovrebbero essere considerati in pazienti con shock e che richiedono multiple dosi di inotropi e/o vasopressori;

- nei pazienti con MIS-C refrattario una seconda dose di IVIG non è sempre raccomandata dato il rischio di sovraccarico di volume e anemia emolitica associata a dosi elevate di IVIG;

- gli steroidi a basso-moderato dosaggio possono essere presi in considerazione anche nei pazienti con forme più lievi di MIS-C che sono persistentemente febbrili e sintomatici nonostante una singola dose di IVIG.

A parere del gruppo di lavoro, essendo emersa una maggiore resistenza dei pazienti con MIS-C al trattamento con le sole IVIG ${ }^{79}$, andrebbe prevista in tutti i casi di MIS-C non critici la combinazione di IVIG e steroide a basse dosi (2 $\mathrm{mg} / \mathrm{kg}$ di metilprednisolone ev per 5 giorni con successivo décalage) come prima linea di trattamento ${ }^{149}$ alla luce anche dei risultati di un recente studio clinico randomizzato ${ }^{150}$.

I farmaci biologici (anakinra, tocilizumab, infliximab) andrebbero presi in considerazione come seconda linea di terapia nei casi non responsivi alle IVIG e ai boli di steroide ${ }^{144,145}$, sulla base di quelle che sono le raccomandazioni previste nella malattia di Kawasaki e di esperienze su alcuni casi con MIS-C ${ }^{149-}$ ${ }^{157}$. La Consensus britannica ${ }^{144}$ non riporta una preferenza nella scelta tra i tre farmaci; quella dell'ACR ${ }^{145}$ fa riferimento all'anakinra per motivi di sicurezza nell'uso del farmaco nei pazienti pediatrici con sindromi iperinfiammatorie e infezione attiva e sull'esperienza in un limitato numero di pazienti con MIS-C riportati in letteratura ${ }^{87,146,152,154}$. L'anakinra (iv o sc) può essere presa in considerazione per il trattamento di MIS-C refrattario a IVIG e glucocorticoidi, in pazienti con MIS-C e caratteristiche della sindrome da attivazione macrofagica (MAS), miocardite con grave deficit di pompa $o$ in pazienti con controindica-

zioni all'uso a lungo termine di glucocorticoidi (Tabella VI).

\section{Misure di supporto}

Le misure di supporto sono riportate nella Tabella VII. I pazienti in stato di shock richiedono il supporto cardiocircolatorio con fluidi e farmaci inotropi; in caso di disfunzione ventricolare si ricorre alla circolazione extracorporea ${ }^{146}$. È raccomandato l'utilizzo di inibitori di pompa protonica in caso di concomitante terapia steroidea e l'avvio di terapia

\section{PROPOSTA DI TRATTAMENTO IMMUNOMODULANTE DELLA MIS-C}

antibiotica ev, nei casi che esordiscono con quadri simil-settici, fino all'esito delle colture ${ }^{14}$.

\section{Gestione dello stato pro-coagulativo}

La MIS-C determina uno stato procoagulativo, con aumento significativo del D-dimero e del fibrinogeno ${ }^{130,131,135}$. L'acido acetilsalicilico è raccomandato a dosaggio antiaggregante $(3-5 \mathrm{mg} /$ $\mathrm{kg} / \mathrm{die})$ nei casi MK-like e/o con interessamento cardiaco e/o trombocito$\mathrm{si}^{145,158}$. La Consensus americana ${ }^{135}$ sugge-
Immunoglobuline

Prima linea di terapia

\section{Glucocorticoidi}

Raccomandati in associazione alla prima linea di terapia in pazienti ad alto rischio secondo il protocollo della malattia di Kawasaki (età < 12 mesi, interessamento cardiaco, alterazioni coronariche ... )

Seconda linea di terapia in caso di fallimento della prima linea di terapia insieme a $2^{\circ}$ bolo di IVIG ev

L'associazione tra IVIG e corticosteroidi (2 $\mathrm{mg} / \mathrm{kg}$ di metilprednisolone) può essere un'opzione da considerare per tutti i casi di $M I S-C$, tenendo in considerazione che in modelli simili (M K) la combinazione dei due trattamenti sembra ridurre il rischio di complicanze

\section{Farmaci biologici}

(anakinra, tocilizumab, infliximab)

Terza linea di terapia: in caso di scarsa risposta alla terapia con IVIG e steroide

Prima/ seconda linea di terapia: in caso di quadro clinico particolarmente severo anche all'esordio (es. segni di MAS, shock o miocardite con grave deficit di pompa) a giudizio clinico
IVIG ev: $2 \mathrm{~g} / \mathrm{kg}$ (calcolo basato sul peso ideale) in unica somministrazione in almeno 12 ore. In caso di paziente con deficit di pompa/ alterazione del bilancio idrico le IVIG devono essere somministrare in almeno 16-24 ore, 0 alternativamente deve essere considerata l'ipotesi di frazionare la dose totale in due somministrazioni.

In caso di inefficacia/ scarsa risposta deve essere considerata l'opportunità di somministrare una seconda dose.

a. Metilprednisolone: $2 \mathrm{mg} / \mathrm{kg} /$ die in $2-3$ somministrazioni per 5 giorni con décalage in 2-3 settimane

b. Metilprednisolone: 10-30 mg/ kg fino a un massimo di $1 \mathrm{~g}$ in bolo una volta al giorno per 1-3 giorni, seguito da metilprednisolone/ prednisone $2 \mathrm{mg} / \mathrm{kg} /$ die con decalage in $2-3$ settimane

La scelta di $\mathbf{a} 0 \mathbf{b}$ va valutata in base alla gravità del quadro clinico e/ o del quadro di laboratorio. In particolare, in caso di elementi clinico/ laboratoristici di sHLH o in caso di shock appare ragionevole il ricorso ai boli di steroide. In caso di segni di coinvolgimento SN C utile considerare l'utilizzo di desametasone (10 $\mathrm{mg} / \mathrm{m}^{2} / \mathrm{die}$ )

Anakinra: $2 \mathrm{mg} / \mathrm{kg} \times 4 /$ die max $100 \mathrm{mg} \mathrm{ev}$ diluito in soluzione fisiologico e somministrato in max $1 \mathrm{~h}$ dalla preparazione) oppure in infusione continua alla dose di $2 \mathrm{mg} / \mathrm{kg}$ dose d'attacco (in bolo) seguita da una dose complessiva fino a $12 \mathrm{mg} / \mathrm{kg} / \mathrm{die}$ (la fiala di anakinra ha una stabilità di circa 6 ore) per una dose massima giornaliera di $400 \mathrm{mg}$.

Anakinra sottocute: 2-6 mg/ kg/ die

Tocilizumab: <30 kg $12 \mathrm{mg} / \mathrm{kg}$ ev; > $30 \mathrm{~kg}$ : $8 \mathrm{mg} / \mathrm{kg}$ ev

Infliximab: $5 \mathrm{mg} / \mathrm{kg} /$ die in $200-500 \mathrm{ml} \mathrm{di}$ soluzione fisiologica in $2 \mathrm{~h} \mathrm{ev}$

Tratto e adattato dal documento del grupppo di studio di Reumatologia pediatrica della SIP (www.sip.it) 


\section{TERAPIE COMPLEMENTARI NELLA MIS-C}

$\checkmark$ Copertura antibiotica ad ampio spettro in attesa del risulta to delle colture (preferire associazione con clindamicina)

$\checkmark$ Inibitore di pompa protonica

$\checkmark$ Supporto cardiovascolare

$\checkmark$ Acido acetilsalicilico (ASA) dosaggio antiaggregante: $3-5 \mathrm{mg} / \mathrm{kg} /$ die in unica somministrazione per os per almeno 6-8 settimane

$\checkmark$ Profilassi/ terapia antitrombotica:

- In pazienti con fattori di rischio tromboembolico o elevazione del D-dimero: Profilassi anti-Trombotica: eparina a basso peso molecolare 2 volte/ die (target anti-X attivato a $4 \mathrm{~h}$ dalla somminisrazione di eparina: 0,2-0,5 $\mathrm{U} / \mathrm{ml}$ ) Per una stratificazione del rischio

- In caso di trombosi venosa o aneurismi delle coronarie*, disfunzione ventricolare sistolica: terapia anti trombotica a giudizio medico/ centro emostasi di riferimento * Per gli aneurismi delle coronarie valgono le indicazioni al trattamento secondo American Heart Association

- In caso di IRA e schistociti, deve essere presa in considerazione l'ipotesi di sindrome Uremicoemolitica acuta/microangiopatia trombotica e l'eventuale utilizzo di eculizumab ev

Tabella VII

\section{FATTORI DI RISCHIO PER TROMBOSI NEI PAZIENTI PEDIATRICI} CON INFEZIONE DA SARS-COV-2

- Catetere venoso centrale; ventilazione meccanica; immobilizzazione prolungata

- 0 besità (BMI $>95^{\circ}$ percentile)

- Tumori attivi, sindrome nefrosica, esacerbazione di fibrosi cistica, crisi vaso-occlusiva falcemica, malattia autoimmune o infiammatoria sottostante

- Cardiopatia congenita o acquisita con stasi venosa o alterato ritorno venoso

- Storia personale o familiare di eventi tromboembolici

- Trombofilia nota (proteina $S$, proteina $C$, deficit di antitrombina, fattore $V$ di Leiden; fattore II G 20210A; anticorpi anti-fosfolipidi)

- Età puberale o post-puberale (>12 anni)

- Terapia estrogenica o estro-progestinica

- Pazienti splenectomizzati per emoglobinopatia

Tabella VIII

risce di iniziare la profilassi antitrombotica nei bambini ricoverati con malattia grave che abbiano almeno un fattore di rischio per trombosi o un dosaggio ematico di D-dimero elevato ( $\geq 5$ volte al valore normale), in assenza di specifiche controindicazioni (Tabella VIII). La stratificazione dei pazienti con MIS-C per il rischio tromboembolico in rapporto al D-dimero è riportata in Tabella $I X$. Nei pazienti instabili o con danno renale è consigliata la terapia eparinica in profilassi in infusione continua ${ }^{135}$. La profilassi antitrombotica è sconsigliata in presenza di trombocitopenia (piastrine $<20.000-50.000 / \mu l)$, ipofibrinogemia (<100 mg/dl), recente sanguinamento maggiore ${ }^{135}$; è indicata dopo la dimissione per un ulteriore periodo che deve essere valutato (ad esempio altri 30 giorni) in caso di persistenza di D-dimero elevato o persistenza di fattori di rischio per trombosi ${ }^{135}$.
In breve. L'obiettivo del trattamento della MIS-C è ridurre l'infiammazione sistemica e ripristinare la funzione d'organo e si basa sul trattamento immunomodulante dell'infiammazione (immunoglobuline, corticosteroidi, farmaci biologici), misure di supporto (trattamento dello shock, terapia antibiotica, gastroprotezione) e gestione dello stato pro-coagulativo.

\section{QUANDO IL PAZIENTE PEDIATRICO SARS-COV-2 POSITIVO VA RICOVERATO IN TERAPIA INTENSIVA?}

La Tabella $X$ riassume la gestione del bambino con sospetto Covid in emergenza.

I criteri di ammissione in Terapia Intensiva Pediatrica (TIP) non differiscono tra pazienti pediatrici positivi o negativi per infezione da SARS-CoV$2^{159,160}$. Nel caso di un paziente SARS-
CoV-2 positivo sarà necessario garantire tutti gli idonei presidi di sicurezza e di isolamento, secondo gli standards e la normativa prevista ${ }^{161}$. Un bambino SARS-CoV-2 positivo potrà richiedere un ricovero in TIP per: 1) le dirette conseguenze della infezione virale, generalmente una grave insufficienza respiratoria; 2) le complicanze dell'infezione (es. MIS-C); 3) per l'insorgen$\mathrm{za}$, in concomitanza dell'infezione da SARS-CoV-2, di altre condizioni che indicano il ricovero.

Il ricovero in TIP è indicato nei casi di insufficienza respiratoria grave come in caso di grave o ingravescente distress respiratorio secondo la definizione dell'OMS (tachipnea con associato almeno uno dei seguenti segni o sintomi: cianosi, ipossia, incapacità di alimentarsi e di idratarsi, letargia, stato di incoscienza o convulsioni) ${ }^{162}$ e di pARDS secondo i criteri della Pediatric Acute Lung Injury Consensus Conference (PALICC): $\mathrm{P} / \mathrm{F} \leq 300$ o $\mathrm{S} / \mathrm{F} \leq 264$ in bilevel o CPAP $\geq 5 \mathrm{~cm} / \mathrm{H}_{2} \mathrm{O}$ ) ${ }^{163}$, per la necessità di monitoraggio continuo dell'efficacia e dell'evoluzione dell'assistenza respiratoria non invasiva, nonché delle eventuali complicanze. Qualora si renda necessaria l'intubazione (indicativamente: $\mathrm{P} / \mathrm{F} \leq 200, \mathrm{~S} / \mathrm{F} \leq 221$, progressivo deterioramento del quadro clinico, ipossia persistente, acidosi respiratoria grave, eccessivo ingombro secretivo) è inevitabile il trasferimento in TIP.

Altre comuni indicazioni al ricovero in TIP sono l'instabilità o l'insufficienza cardiocircolatoria di qualsiasi eziologia non rispondenti al riempimento volemico e/o necessitante di supporto aminico. Si ricorda che, particolarmente nel paziente pediatrico, l'ipotensione è un segno tardivo di insufficienza e che, secondo le linee guida dell'ACCCM sullo shock settico pediatrico, dopo l'iniziale resuscitation bundle (golden hour) il successivo stabilization bundle in TIP richiede un monitoraggio multimodale avanzato $^{164,165}$

L'aspetto emodinamico risulta di particolare rilevanza nella valutazione del paziente con accertata o presunta MIS-C. Nelle forme moderate o gravi la spiccata predisposizione al rapido deterioramento della funzione cardiocircolatoria e il quadro clinico com- 
plesso, variamente caratterizzato da instabilità emodinamica, compromissione respiratoria, alterazione dello stato neurologico, segni di disidratazione, segni di insufficienza renale acuta e dolore addominale grave, possono necessitare di ricovero in ambiente intensivo. A tale proposito, possono facilitare la scelta del setting assistenziale più appropriato la presenza all'ecocardiografia di disfunzione ventricolare sinistra, di anormalità coronariche o di versamento pericardico, oltre all'entità dell'alterazione degli esami di laboratorio (PCR, procalcitonina, IL-6, ferritina, D-dimero, troponina, indici epatici, $\mathrm{LDH})$ e alla rapidità delle loro modificazioni nel tempo ${ }^{81,160,166}$.
STRATIFICAZIONE DEL RISCHIO TROMBOEMBOLICO NEI PAZIENTI CON MIS-C IN RAPPORTO AI VALORI DI D-DIMERO

\begin{tabular}{|c|c|c|c|}
\hline \multirow{4}{*}{ Paziente con MIS-C } & $\begin{array}{l}\text { D-dimero } \\
\geq 5 \text { volte vn }\end{array}$ & $\begin{array}{c}\text { Fattori di rischio } \\
\text { aggiuntivi }\end{array}$ & $\begin{array}{c}\text { Anticoagulazione } \\
\text { suggerita }\end{array}$ \\
\hline & Sì & $\mathrm{No} / \mathrm{nd}$ & Sì \\
\hline & No & Uno o più & Sì \\
\hline & $\mathrm{No}$ & $\mathrm{No}$ & $\mathrm{No}$ \\
\hline
\end{tabular}

Tabella IX
In breve. I criteri di ammissione in TIP non differiscono tra pazienti pediatrici positivi o negativi per l'infezione da SARS-CoV-2 e sono rappresentati dalle dirette conseguenze dell'infezione virale (generalmente una grave insufficienza respiratoria), dalle complicanze dell'infezione (es. MIS-C) e dall'insorgenza, in concomitanza dell'infezione da SARS-CoV-2, di altre condizioni per le quali è indicato il ricovero.

\section{COME VA GESTITO IL PAZIENTE PEDIATRICO CRITICO SARS-COV-2 POSITIVO?}

I pazienti pediatrici ricoverati in TIP vengono assistiti da personale specializzato con un rapporto numerico infermiere/paziente in grado di

\footnotetext{
GESTIONE DEL BAMBINO CON SOSPETTO COVID IN EMERGENZA

$\checkmark$ Come va trattato il bambino che arriva in Pronto Soccorso con sospetto Covid e insufficienza respiratoria acuta ipossiemica?

- In caso di SpO $2<92 \%$ in aria ambiente, prevedere ossigenoterapia con nasocannule $2 \mathrm{l} / \mathrm{min}$.

- Se nonostante l'ossigenoterapia con nasocannule il quadro clinico e la saturazione non migliorano rapidamente, iniziare ossigenoterapia ad alto flusso a FiO 2 incrementali.

Nota bene: si può stimare la $\mathrm{FiO}_{2}$ in maniera approssimativa anche con le cannule nasali $->2-4$ LPM nel bambino e nell'adolescente e 1/2-2 LPM nel lattante e nel bimbo fino ai 2 anni equivalgono a una FiO 2 dello 0,3-0,35 Se la Sat0 2 in paziente con $\mathrm{Hb}$ normale non supera il $90 \%$ si può stimare un $\mathrm{P} / \mathrm{F}<300$. In tale caso siamo praticamente già in condizioni di Acute Lung Injury.

- In caso di mancato miglioramento del quadro clinico nonostante ossigenoterapia ad alto flusso, prevedere CPAP. Nota bene: nel paziente adulto ci sono dati a sostegno di un peggioramento dell'outcome nei pazienti che prolungano una N IV inefficace o con blanda risposta clinica. $\mathrm{N}$ el paziente pediatrico i dati della letteratura non sono così chiaro ma è comunque consigliato non prolungare l'assistenza respiratoria con HFN C se non c'è risposta clinica e strumentale/ emogasanalitica. Un'assistenza con alti flussi e $\mathrm{FiO} 2>0,6$ senza risposta clinica è da considerare come fallita e bisogna passare ad altra modalità di assistenza respiratoria (non invasiva o invasiva)

- Raccomandata intubazione in caso di P/F $\leq 200, S / F \leq 221$, progressivo deterioramento del quadro clinico, ipossia persistente, acidosi respiratoria severa, eccessivo ingombro secretivo

$\checkmark$ Quanto tempo aspetto e come decido se poi inviare o meno al Centro di riferimento dotato di Terapia Intensiva Pediatrica?

In caso di necessità di CPAP nel paziente Covid-positivo in insufficienza respiratoria acuta, è raccomandato il ricovero in Centro di riferimento dotato di TIP.

II ricovero in TIP è raccomandato in caso di:

- Tachipnea con associato almeno uno dei seguenti segni o sintomi: dispnea, elevato W O B, cianosi, ipossia e/ o desaturazione, incapacità di alimentarsi e bere, letargia, stato di incoscienza o convulsioni

- Sindrome da distress respiratorio acuto (P/ F $\leq 300$ o $\mathrm{S} / \mathrm{F} \leq 264$ in bi-level o $\mathrm{CPAP} \geq 5 \mathrm{~cm} / \mathrm{H}_{2} \mathrm{O}$ )

- Necessità di supporto inotropo e/ o monitoraggio emodinamico

- Instabilità, insufficienza cardiocircolatoria o qualsiasi condizione di shock

- Insufficienza multiorgano (MOF)

- Sepsi grave

- Coma

$\checkmark$ Quali eventuali fattori di rischio mi devono indirizzare a un possibile ricovero in Terapia Intensiva Pediatrica?

$\mathrm{N}$ el paziente adulto si stanno affermando score, derivati da studi epidemiologici basati su ampie casistiche internazionali, relativi al rischio di necessità di intubazione e di mortalità del paziente Covid positivo sintomatico.

$\mathrm{N}$ el paziente pediatrico non sono ancora disponibili strumenti simili.

Se pur non vi siano fattori di rischio definiti relativamente al paziente pediatrico Covid sintomatico, sulla base della clinica e delle varie esperienze cliniche si possono considerare come fattori di rischio negativi:

- Presenza all'ecocardiografia di disfunzione ventricolare sinistra di nuova insorgenza, anormalità coronariche o versamento pericardico

- Deterioramento degli esami di laboratorio (PCR, procalcitonina, IL-6, ferritina, D-dimero, troponina, indici epatici, LDH)

- Insufficienza respiratoria progressiva: la mancata riduzione della HR, del RR e del W O B e il mancato miglioramento della Sato 2, a $30^{\prime}$ e a 2 h dall'inizio della terapia con alti flussi sono altamente predittivi di fallimento della $\mathrm{N}$ IV, così come valori elevati di PRISM basale, $\mathrm{CO}_{2}$ e $\mathrm{FiO}_{2}$.
} 
fornire un ottimale trattamento e monitoraggio. La terapia di supporto dell'insufficienza d'organo e di trattamento delle concomitanti patologie si integra con il trattamento atto a soddisfare i bisogni idrico-nutrizionali, a minimizzare la sofferenza, a prevenire infezioni e altre complicanze. Dal punto di vista strutturale andrebbero gestiti preferenzialmente in camere a pressione negativa, se disponibili, $o$ in cui siano presenti almeno 12 ricambi aria/ora. Nel paziente in terapia per patologie concomitanti la terapia non andrebbe sospesa ma eventualmente modificata sulla base dell'evoluzione del quadro clinico complessivo e del rapporto rischio/beneficio legato alla nuova condizione infettiva.

Il numero di pazienti pediatrici ricoverati in TIP in Emilia-Romagna con infezione da SARS-CoV-2 dall'inizio della pandemia è pari a 13 .

Data la limitata numerosità dei pazienti pediatrici con insufficienza respiratoria SARS-CoV-2 correlata, non sono stati definiti indicazioni specifiche al trattamento intensivo che, quindi, seguono i criteri di gradualità progressiva indicati da una Consensus di 27 esperti riferita al Pediatric Lung Injury indipendentemente dall'eziologia $^{167}$. In caso di fallimento delle tecniche di ossigenazione ad alto flusso (HFNC), della CPAP e della ventilazione non invasiva (NIV), si dovrà procedere alla ventilazione invasiva tramite intubazione orotracheale endotracheale (IT). Le raccomandazioni della ESPNIC ${ }^{168}$ per la gestione ventilatoria, anche queste raggiunte tramite consenso di 15 esperti, prediligono le tecniche NIV rispetto alla HFNC, ma tale impostazione non è ripresa dalle ultime linee guida della PICSUK che mettono in discussione questa impostazione volta a un maggior reclutamento alveolare ${ }^{169}$.

Similmente a quanto raccomandato per la gestione del paziente adulto, grande attenzione deve essere rivolta alla protezione del personale durante le manovre a rischio di aerosolizzazione che richiedono presidi di protezione (DPI) di secondo livello. L'utilizzo del videolaringoscopio è consigliato, associato quando possibile a un telo traspa- rente (evita la dispersione dell'aerosol), per consentire di aumentare la distanza tra il paziente e l'operatore (da 16 a $36 \mathrm{~cm})^{169}$; nell'adulto l'uso del videolaringoscopio si associa a riduzione del numero di tentativi infruttuosi. L'uso di tubi cuffiati e del sistema di aspirazione a circuito chiuso riduce il rischio di contaminazione da aerosolizzazione durante le manovre di broncoaspirazione $^{170}$.

Il paziente dovrà essere preossigenato con $\mathrm{O}_{2}$ al $100 \%$ ed è fortemente raccomandato l'utilizzo della rapida sequenza di intubazione (RSI). Le linee guida generali per la ventilazione dei pazienti pediatrici con ARDS possono essere applicate ai pazienti SARS-CoV$2^{171}$. Si consiglia di mantenere un tidal volume di $4-6 \mathrm{ml} / \mathrm{kg}$ di peso ideale e la scelta della PEEP dovrà essere titolata in base alla clinica e ai parametri ventilatori del paziente. Questi pazienti si giovano della pronazione ${ }^{172}$. Nei casi non responsivi è possibile considerare l'utilizzo dell'ossido nitrico (iNO) e della ventilazione oscillatoria ad alta frequenza (HFOV). In caso di shock o ARDS refrattari alla terapia dovrebbe essere preso in considerazione, seguendo rigorosi criteri di selezione, l'ExtraCorporeal Membrane Oxygenation (ECMO).

Nel paziente con instabilità emodinamica, ipoteso con segni di mal perfusione e disidratazione si procederà al riempimento volemico con soluzioni cristalloidi bilanciate $(10-20 \mathrm{ml} / \mathrm{kg}$ in boli ripetuti fino a un $\max 40-60 \mathrm{ml} / \mathrm{kg}$ nella prima ora). Soprattutto in caso di insufficienza respiratoria è consigliabile utilizzare indici di fluid responsiveness e monitoraggio emodinamico avanzato per guidare al meglio il supporto emodinamico senza eccedere nel sovraccarico idrico. In caso di necessità di supporto aminico, adrenalina $\mathrm{e}$ noradrenalina sono di prima scelta ${ }^{173}$, a seconda della presenza o meno di disfunzione sistolica cardiaca. Come farmaci di seconda linea bisogna considerare milrinone, levosimendan e dobutamina. Nei casi di shock non responsivo va valutato l'utilizzo della vasopressina e dei glucocorticoidi.

Nel paziente critico o con sospetta MIS-C bisognerebbe procedere a un'at- tenta valutazione cardiologica (ECG, ecocardiografia, troponina, $\mathrm{LDH}, \mathrm{CPK}$ e CPKMB, pBNP) ed eventualmente procedere a un monitoraggio emodinamico avanzato. In caso di segni di miocardite va considerato l'utilizzo delle IVIG. È suggerito prendere in considerazione l'utilizzo di profilassi con enoxaparina sc soprattutto nei pazienti ad alto rischio di trombosi. Nei quadri di Thrombocytopenia-Associated Multiple Organ Failure (TAMOF) in cui la riduzione della conta piastrinica riflette un processo trombotico microvascolare che può portare a ischemia e disfunzione d'organo (specie insufficienza renale acuta) può essere utile il trattamento di plasma exchange.

In breve. La terapia di supporto dell'insufficienza d'organo e di trattamento delle concomitanti patologie va integrato con il trattamento atto a soddisfare i bisogni idrico-nutrizionali, a minimizzare la sofferenza, a prevenire infezioni e altre complicanze. Nel paziente in terapia per patologie concomitanti la terapia non andrebbe sospesa ma eventualmente modificata sulla base dell'evoluzione del quadro clinico complessivo e del rapporto rischio/beneficio legato alla nuova condizione infettiva.

\section{SONO NECESSARIE MISURE DI SUPPORTO IN MERITO ALLA SALUTE MENTALE DEL BAMBINO E DELL'ADOLESCENTE COVID-19 POSITIVO?}

Il bambino e l'adolescente positivi per SARS-CoV-2 pur essendo, nella stragrande maggioranza dei casi, asintomatici o paucisintomatici, possono vivere con difficoltà il necessario isolamento a domicilio o in ospedale. Aumentano le segnalazioni di un complessivo disagio, anche nei bambini e adolescenti non infetti da SARS-CoV2, conseguenti al periodo di lockdown, alla chiusura delle scuole, alla sospensione dell'attività motoria e alla scarsa socializzazione. La natura e l'entità di questo impatto, difficili da prevedere, dipendono verosimilmente da diversi fattori di vulnerabilità come l'età, le condizioni di salute fisica e mentale preesistenti, le condizioni socioecono- 
miche della famiglia, il più ampio contesto di comunità ${ }^{21,174-179}$.

A questo riguardo non sono comprensibili le recenti indicazioni nazionali per il ritorno allo sport dopo infezione da SARS-CoV-2 sospetta o accertata $^{180}$ : la lista di esami richiesti, indipendentemente dalla valutazione clinica, sembra avere una connotazione di protezione medico-legale più che di ragionevole prudenza, rischia di diventare un ostacolo alla normale ripresa dell'attività fisica, oltre a discostarsi in maniera incomprensibile dalle raccomandazioni di altre agenzie di salute ${ }^{181}$.

Il pediatra di famiglia ha, quindi, un ruolo centrale: sia nel farsi portavoce dei diritti dei suoi assistiti, sia lavorando in rete con la neuropsichiatria dell'infanzia e dell'adolescenza e con la pediatria ospedaliera, sia nel monitorare il fenomeno del disagio conseguente alla pandemia da SARS-CoV-2 in età pediatrica dal punto di vista epidemiologico ${ }^{182}$.

In breve. $\dot{E}$ compito dei professionisti di salute deputati all'assistenza dei bambini e delle famiglie positivi a Covid-19 garantire un adeguato follow-up e un complessivo sostegno, con rassicurazione e rapida reintegrazione all'interno della comunità scolastica (anche nel caso di didattica a distanza) e sociale. $\dot{E}$ necessario rilevare precocemente i segnali di un possibile disagio emotivo, anche familiare, assicurando, se necessario un supporto che veda coinvolte diverse le figure professionali (della Pe- diatria, Neuropsichiatria, del sociale e del mondo educativo-scolastico).

\section{CONCLUSIONI}

Questo documento sintetizza le conoscenze attuali - per lo più basate su consenso di esperti e su studi primari osservazionali retrospettivi - e le esperienze maturate a livello regionale sulla gestione dell'infezione da SARS-CoV-2 nel bambino e nell'adolescente con l'obiettivo di condividere principi di buone prassi. Vista la rapida evoluzione della pandemia sarà utile aggiornarne i contenuti in base alla produzione di nuove evidenze.

Di seguito vengono sottolineati gli aspetti salienti del documento.

- La condivisione fra le professioniste e i professionisti dei criteri di ricovero e di gestione terapeutica dei casi gravi è fondamentale per garantire un approccio equo e basato sulle migliori conoscenze disponibili.

- L'uso profilattico di antibiotici nella gestione dei pazienti pediatrici con Covid-19 non si associa a vantaggi in termini di morbosità e mortalità e concorre allo sviluppo di resistenze antimicrobiche.

- L'attività dei professionisti socio-sanitari deve includere anche la descrizione, gestione e limitazione dei danni psico-fisico-relazionali conseguenti alla pandemia da
SARS-CoV-2 sulla salute di bambini e adolescenti, affetti o meno da Covid-19, e deve poter informare e allertare il livello politico.

- Per le caratteristiche della patologia da Covid-19 in età pediatrica si sottolinea l'importanza di un rafforzamento della rete fra la Pediatria ospedaliera, quella territoriale (sia a livello di Pediatria di famiglia che a livello di Pediatria di comunità e di Neuropsichiatria infantile e adolescenziale), il personale scolastico, educativo, del sociale e la famiglia sia per la gestione strettamente clinica che per la riduzione del disagio, con priorità nei bambini di famiglie a maggiore fragilità.

- Per quel che riguarda i casi pediatrici ricoverati, vista la scarsità di dati disponibili in letteratura, sarebbe opportuno che le Pediatrie identifichino un set di variabili da monitorare nel tempo utilizzando una metodologia e definizioni condivise.

\section{Conflitto di interesse: nessuno}

\section{Indirizzo per corrispondenza:}

Susanna Esposito

susannamariaroberta.esposito@unipr.it

\section{*GRUPPO DI LAVORO SU COVID-19 IN PEDIATRIA DELLA REGIONE EMILIA-ROMAGNA (RE-CO-PED)}

G iuseppe Cannalire, Maria Sole Magistrali, Raymond Akamin, Ospedale Guglielmo da Saliceto, Piacenza; Icilio Dodi, Alberto Argentiero, Luciana Abate, Claudia De Guido, Umberto Fanelli, Marco Pappalardo, Francesco Pecora, Francesco Sogni, Azienda Ospedaliero-Universitaria, Università di Parma; Giancarlo Gargano, Alessandro Volta, Costantino Panza, Sara Fornaciari, Giovanna Russo, AUSL-IRCCS di Reggio Emilia; Barbara Predieri, Azienda Ospedaliero-Universitaria, Università di Modena e Reggio Emilia; Jennifer Chiarolanza, AUSL Modena; La ura Andreozzi, Marianna Fabi, UO di Pediatria d'Urgenza, IRCCS Azienda Ospedaliero-Universitaria, Policlinico di S. Orsola, Bologna; Fabrizio Sandri, Chiara G hizzi, Rita Ricci, Luciana N icoli, Alessandro Ballestrazzi, Tiziano Dall'O sso, AUSL Bologna; Laura Serra, AUSL Imola; Claudia Guiducci, Alessandra lacono, Anna Maria Magistà, AUSL della Romagna, Ravenna; Cristina Malaventura, Adriana Fumarola, Azienda Ospedaliero-Universitaria, Università di Ferrara; Marcello Bergamini, AUSL Ferrara; Enrico Valletta, Marcello Stella, Franco Mazzini, AUSL della Romagna, Forli-Cesena; G ina Ancora, Giovanni Pancaldi, Beatrice Scarpellini, Cristina Ferrè, AUSL della Romagna, Rimini; Rossana De Palma, Simona Di Mario, Fabia Franchi, Roberta Giroldini, Anna Maria Marata, Giovanna Mattei, Valentina Solfrini, DG Cura della Persona, Salute e Welfare - Regione Emilia-Romagna; M aria Luisa M oro, Agenzia Sanitaria e Sociale della Regione Emilia-Romagna. 


\section{Bibliografia}

1. Zhu N, Zhang D, Wang W, et al. China Novel Coronavirus Investigating and Research Team. A Novel Coronavirus from Patients with Pneumonia in China, 2019. N Engl J Med 2020;382:727-33.

2. Bialek S, Gierke R, Hughes M, McNamara LA, Pilishvili T, Skoff T. Coronavirus Disease 2019 in Children - United States, February 12 - April 2, 2020. MMWR Morb Mortal Wkly Rep. 2020;69:422-6.

3. Castagnoli R, Votto M, Licari A, et al. Severe Acute respiratory syndrome coronavirus 2 (SARSCoV-2) infection in children and adolescents: A systematic review. JAMA Pediatr 2020;174:882-9.

4. Dong Y, Dong Y, Mo X, et al. Epidemiology of Covid-19 among children in China. Pediatrics 145(6):e20200702.

5. Götzinger F, Santiago-García B, NogueraJulián A, at al. Covid-19 Study Group. Covid19 in children and adolescents in Europe: a multinational, multicentre cohort study. Lancet Child Adolesc Heal 2020;4:653-61.

6 . Verity R, Okell LC, Dorigatti I, et al. Estimates of the severity of coronavirus disease 2019 . a model-based analysis. Lancet Infect Dis 2020;20:669-77.

7. Patel KP, Vunnam SR, Patel PA, et al. Transmission of SARS-CoV-2: an update of current literature. Eur J Clin Microbiol Infect Dis 2020;39:2005-11.

8. Wölfel R, Corman VM, Guggemos W, et al Virological assessment of hospitalized patients with Covid-2019. Nature. 2020;581: 465-9.

9. European Centre for Disease Prevention and Control. Covid-19 testing strategies and objectives. 15 September 2020. ECDC: Stockholm; 2020. https://www.ecdc.europa. eu/en/publications-data/covid-19-testing-strategies-and-objectives. Ultimo accesso: 2 febbraio 2021 .

10. Ludvigsson JF. Systematic review of Covid-19 in children shows milder cases and a better prognosis than adults. Acta Paediatr 2020;109:1088-95.

11. Deville JG. Coronavirus disease 2019 (Covid-19): Clinical manifestations and diagnosis in children. UpToDate aggiornato 19.01.2021 https://www.uptodate.com/contents/coronavirus-disease-2019-covid-19-clinical-manifestations-and-diagnosis-in-children?topicRef= 127454\&source=see_link. Ultimo accesso: 2 febbraio 2021.

12. Rapporto ISS Covid-19 n. 63/2020 - Apertura delle scuole e andamento dei casi confermati di SARS-CoV-2: la situazione in Italia. Versione del 30 dicembre 2020.

13. European Centre for Disease Prevention and Control. Covid-19 in children and the role of school settings in transmission - first update (23 december). Stockholm, 2020.

14. Li X, Xu W, Dozier M, et al.; UNCOVER. The role of children in transmission of SARS-CoV-2 A rapid review. J Glob Health 2020;10: 011101. 15. Munro APS, Faust SN. Children are not Covid-19 super spreaders: time to go back to school. Arch Dis Child 2020;105:618-9.
16. Hsieh KY, Kao WT, Li DJ, et al. Mental health in biological disasters: From SARS to Covid-19. Int J Soc Psychiatry 2020, Jul 29 [Epub ahead of print]

17. Esposito S, Giannitto N, Squarci A, et al. Development of psychological problems among adolescents during school closures because of the Covid-19 lockdown phase in Italy: a cross-sectional survey. Front Pediatr 2021, Jan 22 [Epub ahead of print]

18. de Miranda DM, da Silva Athanasio B, Oliveira ACS, Simoes-e-Silva AC. How is Covid19 pandemic impacting mental health of children and adolescents? Int J Disaster Risk Reduct 2020;51:101845.

19. Imai N, Gaythorpe KAM, Abbott S,et al. Adoption and impact of non-pharmaceutical interventions for Covid-19. Wellcome Open Res 2020;5:59.

20. Viner RM, Russell SJ, Croker H, et al. School closure and management practices during coronavirus outbreaks including Covid19: a rapid systematic review. Lancet Child Adolesc Health 2020;4:397-404

21. Singh S, Roy D, Sinha K, Parveen S, Sharma G, Joshi G. Impact of Covid-19 and lockdown on mental health of children and adolescents: A narrative review with recommendations. Psychiatry Res 2020;293:113429.

22. ECDC. Covid-19 in children and the role of school settings in transmission - first update. https://www.ecdc.europa.eu/en/publications-data/children-and-school-settings-covid19-transmission. Ultimo accesso: 2 febbraio 2021.

23. Centers for Disease Control and Prevention. Coronavirus Disease 2019 (Covid-19). https://www.cdc.gov/coronavirus/2019 nCoV/index.html. Ultimo accesso: 12 dicembre 2020 .

24. World Health Organization. https://www. who.int/emergencies/diseases/novelcoronavirus- 2019/advice-for-public. Ultimo accesso: 12 dicembre 2020.

25 Ministero della Salute. http://www.salute. gov.it/portale/nuovocoronavirus/homeNuovoCoronavirus.jsp. Ultimo accesso: 12 dicembre 2020 .

26. Cheng KK, Lam TH, Leung CC. Wearing face masks in the community during the $\mathrm{Co}$ vid-19 pandemic: altruism and solidarity. Lancet 2020, Apr 16 [Epub ahead of print].

27. Esposito S, Principi N, Leung CC, Migliori GB. Universal use of face masks for success against Covid-19: evidence and implications for prevention policies. Eur Respir J 2020;55 2001260.

28. Hoang A, Chorath K, Moreira A, et al. Covid-19 in 7780 pediatric patients: A systematic review. E Clinical Medicine 2020;24:100433.

29. Centers for Disease Control and Prevention "information for pediatric heath care providers" www.cdc.gov/coronavirus/2019/ ncov/hcp/pediatric-hcp.html. Ultimo accesso: 12 dicembre, 2020

30. Kuttiatt VS, Abraham PR, Menon RP, Vaidya PC, Rahi M. Coronavirus disease 2019 in children: Clinical \& epidemiological implications. Indian J Med Res 2020;152:21-40.
31. Son MBF. Coronavirus disease 2019 (Covid-19): Multisystem inflammatory syndrome in children (MIS-C) clinical features, evaluation, and diagnosis. UpToDate aggiornato 25.09.2020 https://www.uptodate.com/contents/coronavirus-disease-2019-covid-19-multisystem-inflammatory-syndrome-in-childrenmis-c-clinical-features-evaluation-and-diagnosis. Ultimo accesso: 2 febbraio 2021.

32. Circolare Ministero Salute 29 maggio 2020 - Ricerca e gestione dei contatti di casi Covid19 (Contact tracing) e App Immuni. https:// www.trovanorme.salute.gov.it/norme/ren derNormsanPdf?anno $=2020 \& \operatorname{codLeg}=74178 \&$ parte $=1 \% 20 \&$ serie $=$ null. Ultimo accesso: 2 febbraio 2021.

33. Huff HV, Singh A. Asymptomatic transmission during the Covid-19 pandemic and implications for public health strategies. Clin Infect Dis 2020, May 28 [Epub ahead of print].

34. Circolare Ministero Salute 8 gennaio 2021 Aggiornamento della definizione di caso Covid19 e strategie di testing. https://www.trovanorme.salute.gov.it/norme/renderNormsanPdf?a nno $=2021 \& \operatorname{codLeg}=78155 \&$ parte $=1 \% 20 \&$ se rie=null. Ultimo accesso: 2 febbraio 2021 .

35. Lin EE, Blumberg TJ, Adler AC, et al. Incidence of Covid-19 in Pediatric Surgical Patients Among 3 US Children's Hospitals. JAMA Surg 2020; 155:775-777.

36. DECRETO-LEGGE 14 gennaio 2021, n. 2 Ulteriori disposizioni urgenti in materia di contenimento e prevenzione dell'emergenza epidemiologica da Covid-19 e di svolgimento delle elezioni per l'anno 2021. https://www. gazzettaufficiale.it/eli/id/2021/01/14/21G00 002/sg. Ultimo accesso: 1 febbraio 2021. 37. Wang W, Xu Y, Gao R, et al. Detection of SARS-CoV-2 in Different Types of Clinical Specimens. JAMA 2020;323:1843.

38. Centers for Disease Control and Prevention. Interim Guidelines for Collecting, Handling, and Testing Clinical Specimens for Covid-19. https://www.cdc.gov/coronavirus/ 2019 ncov/lab/guidelines-clinical-specimens.html. Ultimo accesso: 12 dicembre 2020.

39. Donà $\mathrm{D}$, Minotti $\mathrm{C}$, Costenaro $\mathrm{P}, \mathrm{Da}$ Dalt L, Giaquinto C. Fecal-oral transmission of SARSCov- 2 in children. Pediatr Infect Dis J 2020;39:e133-e134

40. Palmas G, Moriondo M, Trapani S, et al. Nasal Swab as Preferred Clinical Specimen for Covid- 19 Testing in Children. Pediatr Infect Dis J 2020;39:e267-e270.

41. Capecchi E, Di Pietro GM, Luconi E; Testing Pediatric Covid-19 (TPC-19). Is Nasopharyngeal Swab Comparable With $\mathrm{Na}$ sopharyngeal Aspirate to Detect SARS-CoV-2 in Children? Pediatr Infect Dis J 2020;39:e288e289.

42. Circolare Ministero Salute 12 ottobre 2020 - Trasmissione documento "Prevenzione e risposta a Covid-19: evoluzione della strategia e pianificazione nella fase di transizione per il periodo autunno-invernale. https://www.trovanorme.salute.gov.it/norme/renderNormsanPdf?anno $=2020 \&$ codLeg $=76597 \&$ parte $=1 \%$ 20\&serie=null. Ultimo accesso: 1 febbraio 2021. 
43. Russo A, Minichini C, Starace M, Astorri R, Calò F, Coppola N; Vanvitelli Covid-19 group. Current Status of Laboratory Diagnosis for Covid-19: A Narrative Review. Infect Drug Resist 2020;13:2657-65.

44. CDC. Overview of Testing for SARS-CoV2 (Covid-19). Centers for Disease Control and Prevention, Atlanta, 2020. Available from: https://www.cdc.gov/coronavirus/2019ncov/hcp/testingoverview. htlm. Ultimo accesso: 12 dicembre 2020.

45. Dinnes J, Deeks JJ, Adriano A, Berhane S, et al. Cochrane Covid-19 Diagnostic Test Accuracy Group. Rapid, point-of-care antigen and molecular-based tests for diagnosis of SARSCoV-2 infection. Cochrane Database Syst Rev 2020;8:CD013705.

46. Paltiel AD, Zheng A, Walensky RP. Assessment of SARS-CoV-2 Screening Strategies to Permit the Safe Reopening of College Campuses in the United States. JAMA Netw Open 2020;3:e2016818.

47. Watson J, Whiting PF, Brush JE. Interpreting a Covid-19 test result. BMJ 2020;369: m1808.

48. Smith-Norowitz TA, Hammerschlag MR, Kohlhoff S. Coronavirus Disease 2019 (Covid19) infection rates in a private school in Brooklyn, New York. Acta Paediatr 2021, Feb 1 [Epub ahead of print].

49. Pecora ND, Zand MS. Measuring the Serologic Response to Severe Acute Respiratory Syndrome Coronavirus 2: Methods and Meaning. Clin Lab Med 2020;40:603-14.

50. Van Elslande J, Decru B, Jonckheere S, et al. Antibody response against SARS-CoV-2 spike protein and nucleoprotein evaluated by four automated immunoassays and three ELISAs. Clin Microbiol Infect 2020;26:1557.e1-1557.e7.

51. Chan K, Beck C, Chauvin-Kimoff L, et al.; Acute Care Committee POSITION STATEMENT of The Canadian Paediatric Society. The acute management of paediatric coronavirus disease 2019 (Covid-19). Available from: https://www.cps.ca/en/documents/position/the-acute-management-of-paediatric-coronavirus-disease-2019covid-19 Ultimo accesso: 12 dicembre 2020 .

52. Venturini E, Montagnani C, Garazzino S, et al. Italian SITIP-SIP SARS-Cov-2 pediatric infection study group. Treatment of children with Covid-19: position paper of the Italian Society of Pediatric Infectious Disease. Ital J Pediatr 2020;46:139.

53. Garazzino $\mathrm{S}$, Montagnani $\mathrm{C}$, Donà $\mathrm{D}$, et al. Italian SITIP-SIP Pediatric Infection Study Group; Italian SITIP-SIP SARS-CoV-2 paediatric infection study group. Multicentre Italian study of SARS-CoV-2 infection in children and adolescents, preliminary data as at 10 April 2020. Euro Surveill 2020;25:2000600.

54. Vergine G, Fantini M, Marchetti F, et al. Regione Emilia-Romagna Covid-19 Pediatric Working Group (RERCOPed). Home Management of Children With Covid-19 in the Emilia-Romagna Region, Italy. Front Pediatr 2020;8:575290.

55. Zhang Q, Bastard P, Liu Z, et al. Inborn errors of type I IFN immunity in patients with li- fe-threatening Covid-19. Science 2020;370: eabd 4570

56. Marlais M, Wlodkowski T, Vivarelli M, et al. The severity of Covid-19 in children on immunosuppressive medication. Lancet Child Adolesc Health 2020;4:e17-e18.

57. Esposito S; Parma Covid-19 Pediatric Working Group (PaCoPed), Voccia E, et al. Telemedicine for management of paediatric infectious diseases during Covid-19 outbreak. J Clin Virol 2020;129:104522.

58. Paget J, Caini S, Cowling B, et al. The impact of influenza vaccination on the Covid-19 pandemic? Evidence and lessons for public health policies. Vaccine 2020;38:6485-6.

59. Nicastro E, Di Giorgio A, Zambelli M, et al. Impact of the Severe Acute Respiratory Syndrome Coronavirus 2 Outbreak on Pediatric Liver Transplant Recipients in Lombardy, Northern Italy. Liver Transpl 2020;26:1359-62. 60. Arrigo S, Alvisi P, Banzato C, et al. Management of paediatric IBD after the peak of $\mathrm{Co}^{-}$ vid-19 pandemic in Italy: A position paper on behalf of the SIGENP IBD working group. Dig Liver Dis 2021;53(2):183-9.

61. Filocamo G, Minoia F, Carbogno S, Costi S, Romano M, Cimaz R; Pediatric Rheumatology Group of the Milan Area. Absence of severe complications from SARS-CoV-2 infection in children with rheumatic diseases treated with biologic drugs. J Rheumatol 2020, Apr 25 [Epub ahead of print].

62. Koker O, Demirkan FG, Kayaalp G, et al. Does immunosuppressive treatment entail an additional risk for children with rheumatic diseases? A survey-based study in the era of Covid-19. Rheumatol Int 2020;40:1613-23.

63. Vasudevan A, Mantan M, Krishnamurthy $\mathrm{S}$, et al. and the Indian Society of Pediatric Nephrology. Managing children with renal diseases during the Covid-19 pandemic. Indian Pediatr 2020;57:641-651.

64. Abrams EM, Szefler SJ. Manging asthma during coronavirus disease-2019: An example for other chronic conditions in children and adolescents. J Pediatr 2020;222:221-6.

65. Bezzerri V, Lucca F, Volpi S, Cipolli M. Does cystic fibrosis constitute an advantage in Covid-19 infection? It J Pediatr 2020;46: 143. 66. Balduzzi A, Brivio E, Rovelli A, et al. Lessons after the early management of the Covid19 outbreak in a pediatric transplant ad hemato-oncology center embedded within a Covid19 dedicated hospital in Lombardia, Italy. Estote parati. Bone Marrow Transplant 2020;55:1900-1905.

67. Laventhal NT, Graham RJ, Rasmussen SA, Urion DK, Kang PB. Ethical decision-making for children with neuromuscular disorders in the Covid-19 crisis. Neurology 2020;95:260265.

68. Barron E, Bakhai C, Kar P, et al. Associations of type 1 and type 2 diabetes and Covid19- related mortality in England: a whole population study. Lancet Diabetes Endocrinol 2020;11:965.

69. Selvin E, Juraschek SP. Diabetes Epidemiology in the Covid-19 Pandemic. Diabetes Care 2020;43:1690-4.
70. Kamrath CM, Mönkemöller K, Biester T, et al. Ketoacidosis in Children and Adolescents With Newly Diagnosed Type 1 Diabetes During the Covid-19 Pandemic in Germany. JAMA 2020;324:801-3.

71. Predieri B, Leo F, Candia F, et al. Glycemic Control improvement in Italian Children and Adolescents with Type 1 Diabetes followed through Telemedicine during Lockdown due to the Covid-19 Pandemic. Fronts Endocrinol 2020;11:965.

72. Bornstein SR, Rubino F, Khunti K, et al. Personal View Practical recommendations for the management of diabetes in patients with Covid-19. Lancet Diabetes Endocrinol 2020; 8:546-50.

73. Tresoldi AS, Sumilo D, Perrins M, et al. Increased infection risk in Addison's disease and congenital adrenal hyperplasia. J Clin Endocrinol Metab 2020;105:418-29.

74.Hahner S, Spinnler C, Fassnacht M, et al. High incidence of adrenal crisis in educated patients with chronic adrenal insufficiency: a prospective study. J Clin Endocrinol Metab 2015;100:407- 16 .

75. Bornstein SR, Allolio B, Arlt W, et al. Diagnosis and treatment of primary adrenal insufficiency: an Endocrine Society Clinical Practice Guideline. J Clin Endocrinol Metab 2016; 101:364-89.

76. Royal College of Pediatrics and Child Health. Guidance: Paediatric multisystem inflammatory syndrome temporally associated with Covid-19. https://www.rcpch.ac.uk/resources/guidancepaediatric-multisystem-inflammatory-syndrometemporallyassociatedcovid-19. Ultimo accesso: 12 dicembre 2020. 77. Center for Disease Control and Prevention. Multisystem Inflammatory Syndrome in Children (MIS-C). https://www.cdc.gov/ mis-c. Ultimo accesso: 12 dicembre 2020. 78. European Centre for Disease Prevention and Control. Paediatric inflammatory multisystem syndrome and SARS-CoV-2 infection in children - 15 May 2020. ECDC: Stockholm. Ultimo accesso: 12 dicembre 2020.

79. Verdoni L, Mazza A, Gervasoni A, et al. An outbreak of severe Kawasaki-like disease at the Italian epicentre of the SARSCoV- 2 epidemic: an observational cohort study. Lancet 2020;6736:1-8.

80. Riphagen S, Gomez X, Gonzalez-Martinez C, Wilkinson N, Theocharis P. Hyperinflammatory shock in children during Covid-19 pandemic. Lancet 2020;395:1607-8.

81. World Health Organization. Multisystem inflammatory syndrome in children and adolescents with Covid-19. Published May 15, 2020. https://www.who.int/newsroom/ commentaries/detail/multisystem-inflammatorysyndrome-in-children-and-adolescents-withcovid- 19 Ultimo accesso: 12 dicembre 2020. 82. Radia T, Williams N, Agrawal P, et al. Multi-system inflammatory syndrome in children \& adolescents (MIS-C): A systematic review of clinical features and presentation. Paediatr Respir Rev 2020;S1526-0542(20)30117-2. 83. Greene AG, Saleh M, Roseman E, Sinert $\mathrm{R}$. Toxic shock-like syndrome and Covid-19: a 
case report of multisystem $\mathrm{m}$ inflammatory syndrome in children (MIS-C). Am J Emerg Med 2020;1207:5-6.

84. Chiotos K, Bassiri H, Behrens EM, et al. Multisystem inflammatory syndrome in children during the Covid-19 pandemic: a case series. J Pediatr Infect Dis 2020;9:393-8.

85. Toubiana J, Poirault C, Corsia A, et al Kawasaki-like multisystem inflammatory syndrome in children during the Covid-19 pandemic in Paris, France: prospective observational study. Br Med J 2020;369: 369:m2094. 86. Cheung EW, Zachariah P, Gorelik M, et al. Multisystem inflammatory syndrome related to Covid-19 in previously healthy children and adolescents in New York city. JAMA 2020; 324:294-6.

87. Whittaker E, Bamford A, Kenny J, et al.for the PIMS-TS Study Group and EUCLIDS and PERFORM Consortia. Clinical characteristics of 58 children with a pediatric inflammatory multisystem syndrome temporally associated with SARS-CoV-2. JAMA 2020;324:259-69.

88. Grimaud M, Starck J, Levy M, et al. Acute myocarditis and multisystem inflammatory emerging disease following SARS-CoV-2 infection in critically ill children. Ann Intensive Care 2020;10:69.

89. Ahmed M, Advani S, Moreira A, et al. Multisystem inflammatory syndrome in children: A systematic review. EClinicalMedicine 2020; 26:100527.

90. Waltuch T, Gill P, Zinns LE, et al. Features of Covid-19 post-infectious cytokine release syndrome in children presenting to the emergency department. Am J Emerg Med 2020;38: 2246.e3-2246.e6.

91. Ramcharan T, Nolan O, Lai CY, et al. Paediatric inflammatory multisystem syndrome: temporally associated with SARSCoV-2 (PIMS-TS): cardiac features, management and short-term outcomes at a UK tertiary paediatric hospital. Pediatr Cardiol 2020;41:1391401.

92. Pouletty M, Borocco C, Ouldali N, et al. Paediatric multisystem inflammatory syndrome temporally associated with SAR-SCoV- 2 mimicking Kawasaki disease (Kawa-Covid19): a multicentre cohort. Ann Rheum Dis 2020;79:999-1006.

93. Kaushik S, Aydin SI, Derespina KR, et al. Multisystem inflammatory syndrome in children (MIS-C) associated with SARSCoV- 2 infection: a multi-institutional study from New York City. J Pediatr 2020;224:24-9.

94. Dufort EM, Koumans EH, Chow EJ, et al. for the New York State and Centers for Disease Control and Prevention Multisystem Inflammatory Syndrome in Children Investigation Team. Multisystem inflammatory syndrome in children in New York state. N Engl J Med 2020;383:347-58.

95. Feldstein LR, Rose EB, Horwitz SM, et al. for the Overcoming Covid-19 Investigators, and the CDC Covid-19 Response Team. Multisystem inflammatory syndrome in U.S. children and adolescents. N Engl J Med 2020;383: 334-46. 96. Belhadjer Z, Méot M, Bajolle F, et al. Acute heart failure in multisystem inflammatory syn- drome in children (MIS-C) in the context of global SARS-CoV-2 pandemic. Circulation 2020;142:429-36.

97. Godfred-Cato S, Bryant B, Leung J, et al. Covid-19-Associated Multisystem Inflammatory Syndrome in Children - United States, March-July 2020. MMWR Morb Mortal Wkly Rep 2020;69:1074-80.

98. Davies P, Evans C, Kanthimathinathan $\mathrm{HK}$, et al. Intensive care admissions of children with paediatric inflammatory multisystem syndrome temporally associated with SARS-CoV-2 (PIMSTS) in the UK: a multicentre observational study. Lancet Child Adolesc Health 2020;4:669-77.

99. Lee PY, Day-Lewis M, Henderson LA, et al. Distinct clinical and immunological features of SARS-CoV-2-induced multisystem inflammatory syndrome in children. J Clin Invest 2020;130:5942-50.

100. Deza Leon MP, Redzepi A, McGrath E, et al. Covid-19-Associated Pediatric Multisystem Inflammatory Syndrome. J Pediatric Infect Dis Soc 2020;9:407-8.

101. Rauf A, Vijayan A, John ST, Krishnan R, Latheef A. Multisystem Inflammatory Syndrome with Features of Atypical Kawasaki Disease during Covid-19 Pandemic. Indian J Pediatr 2020;87:745-7.

102. Ravelli A, Minoia F, Davì S, et al. Paediatric Rheumatology International Trials Organisation; Childhood Arthritis and Rheumatology Research Alliance; Pediatric Rheumatology Collaborative Study Group; Histiocyte Society. 2016 Classification Criteria for $\mathrm{Ma}$ crophage Activation Syndrome Complicating Systemic Juvenile Idiopathic Arthritis: A European League Against Rheumatism/ American College of Rheumatology/Paediatric Rheumatology International Trials Organisation Collaborative Initiative. Ann Rheum Dis. 2016;75:481-9.

103. AIFA. Principi di gestione dei casi di $\mathrm{Co}-$ VID-19 nel setting domiciliare. https://www. aifa.gov.it/documents/20142/1269602/SOC territoriale_09.12.2020.pdf/0f4896f4-1eac32e2-bc26-5f5d279492b4. Ultimo accesso: 1 febbraio 2021.

104. European Medicines Agency. EMA gives advice on the use of non-steroidal antiinflammatories for Covid-19. Available from: https://www.ema.europa.eu/en/news/ema-givesadvice- use-non-steroidal-anti-inflammatories-covid-19. Ultimo accesso: 21 aprile 2020. 105. WHO. The use of non-steroidal anti-inflammatory drugs (NSAIDs) in patients with Covid19. Available from: https://www.who. int/publications/i/item/the-use-of-non-steroidal-antiinflammatory-drugs-(nsaids)-in-patients-withcovid-19. Ultimo accesso: 21 aprile 2020.

106. Martins-Filho PR, do Nascimento-Júnior EM, Santos VS. No current evidence supporting risk of using Ibuprofen in patients with Covid-19. Int J Clin Pract 2020;74:e13576.

107. Sodhi M, Etminan M. Safety of Ibuprofen in Patients With Covid-19: Causal or Confounded? Chest 2020;158:55-6.

108. AIFA. Trattamenti utilizzabili nei pazienti CoVID-19 nel setting ospedaliero. https:// www.aifa.gov.it/documents/20142/1269602/ SOC_ospedaliera_09.12.2020.pdf/021a4ffe7a80-32ed-ee9c-65a383ff1b47. Ultimo accesso: 1 febbraio 2021.

109. WHO. Modes of transmission of virus causing Covid-19: implication for IPC precaution recommendation. World Health Organization 2020. Available from: https://www. who.int/newsroom/commentaries/detail/ modes-of-transmission-of-virus-causing-covid19-implications-for-ipcprecaution-recommendations. Ultimo accesso: 21 aprile 2020.

110. Leis JA, Born KB, Theriault G, Ostrow O, Grill A, Johnston KB. Using antibiotics wisely for respiratory tract infection in the era of $\mathrm{Co}^{-}$ vid-19. BMJ 2020;371:m4125.

111. Langford BJ, So M, Raybardhan S, et al. Bacterial co-infection and secondary infection in patients with Covid-19: a living rapid review and meta-analysis. Clin Microbiol Infect 2020;26:1622-9.

112. Furtado RHM, Berwanger O, Fonseca HA, et al; COALITION Covid-19 Brazil II Investigators. Azithromycin in addition to standard of care versus standard of care alone in the treatment of patients admitted to the hospital with severe Covid-19 in Brazil (COALITION II): a randomised clinical trial. Lancet 2020;396:959-67.

113. Oldenburg CE, Doan T. Azithromycin for severe Covid-19. Lancet 2020;396:936-7.

114. Lamontagne F, Agoritsas T, Macdonald $\mathrm{H}$, et al. A living WHO guideline on drugs for Covid-19. BMJ 2020;370:m3379.

115. WHO Rapid Evidence Appraisal for Covid-19 Therapies (REACT) Working Group, Sterne JAC, Murthy S, Diaz JV, et al. Association Between Administration of Systemic Corticosteroids and Mortality Among Critically Ill Patients With Covid-19: A Meta-analysis. JAMA 2020;324:1330-41.

116. RECOVERY Collaborative Group, Horby P, Lim WS, Emberson JR, et al. Dexamethasone in Hospitalized Patients with Covid-19 Preliminary Report. N Engl J Med 2020, Jul 17 [Epub ahead of print].

117. WHO. Corticosteroids for Covid-19. Living guidance. World Health Organization 2020. Available from: https://apps.who.int/ iris/handle/10665/334125. Ultimo accesso 21 aprile 2020.

118. AIFA. Corticosteroidi nella terapia dei pazienti adulti con Covid-19. https://www.aifa gov.it/documents/20142/1123276/Corticosteroidi_06.10.2020.pdf/075c9302-895c-4d7e$11 \mathrm{bc}-0 \mathrm{e} 2319082 \mathrm{ffc}$. Ultimo accesso: 1 febbraio 2021.

119. Parr JB. Time to Reassess Tocilizumab's Role in Covid-19 Pneumonia. JAMA Intern Med 2021;181(1):12-5.

120. Hermine O, Mariette X, Tharaux PL, Resche-Rigon M, Porcher R, Ravaud P; CORIMUNO-19 Collaborative Group. Effect of Tocilizumab vs Usual Care in Adults Hospitalized With Covid-19 and Moderate or Severe Pneumonia: A Randomized Clinical Trial. JAMA Intern Med 2021;181(1):32-40.

121. Pain CE, Felsenstein S, Cleary G, et al. Novel paediatric presentation of Covid-19 with 
ARDS and cytokine storm syndrome without respiratory symptoms. Lancet Rheumatol 2020;2:e376-e379.

122. Chen P, Nirula A, Hellen R et al, BLAZE1 Investigators. SARS-CoV-2 Neutralizing Antibody LY-CoV555 in Outpatients with Covid19. N Engl J Med 2021;384(3):229-237. doi: 10.1056/NEJMoa2029849.

123. Gottlieb RL, Nirula A, Chen P, et al. Effect of Bamlanivimab as Monotherapy or in Combination With Etesevimab on Viral Load in Patients With Mild to Moderate Covid-19: A Randomized Clinical Trial. JAMA 2021; Epub Jan 21. e210202. doi: 10.1001/jama.2021.0202.

124. AIFA- Parere CTS su anticorpi monoclonali: https://www.aifa.gov.it/documents/ 20142/1289678/parere_cts_monoclonali_04.0 2.2021.pdf/68737075-6f07-2a43-7f940bc55f2e38f1.

125. Boulware DR, Pullen MF, Bangdiwala AS, et al. A Randomized Trial of Hydroxychloroquine as Postexposure Prophylaxis for Covid-19. N Engl J Med 2020;383:517-25.

126. Mitjà $\mathrm{O}$, Corbacho-Monné $\mathrm{M}$, Ubals $\mathrm{M}$, et al; BCN-PEP-CoV2 Research Group. A Cluster- Randomized Trial of Hydroxychloroquine for Prevention of Covid-19. N Engl J Med 2021;384(5):417-27.

127. Fiolet T, Guihur A, Rebeaud ME, Mulot M, Peiffer-Smadja N, Mahamat-Saleh Y. Effect of hydroxychloroquine with or without azithromycin on the mortality of coronavirus disease 2019 (Covid-19) patients: a systematic review and meta-analysis. Clin Microbiol Infect 2021;27(1):19-27.

128. WHO Solidarity Trial Consortium. Repurposed Antiviral Drugs for Covid-19 - Interim WHO Solidarity Trial Results. N Engl J Med 2020, Dec 2 [Epub ahead of print].

129. Simonovich VA, Burgos Pratx LD, Scibona P, et al. PlasmAr Study Group. A Randomized Trial of Convalescent Plasma in Covid-19 Severe Pneumonia. N Engl J Med 2020, Nov 24 [Epub ahead of print].

130. Barnes GD, Burnett A, Allen A, et al. Thromboembolism and anticoagulant therapy during the Covid-19 pandemic: interim clinical guidance from the anticoagulation forum. J Thromb Thrombolysis 2020;50:72-81.

131. Al-Ghafry M, Aygun B, Appiah-Kubi A, et al. Are children with SARS-CoV-2 infection at high risk for thrombosis? Viscoelastic testing and coagulation profiles in a case series of pediatric patients. Pediatr Blood Cancer 2020;67: e28737.

132. Loi M, Branchford B, Kim J, Self C, Nuss R. Covid-19 anticoagulation recommendations in children. Pediatr Blood Cancer 2020; 67:e28485.

133. Bhattacharyya R, Iyer P, Phua GC, Lee $\mathrm{JH}$. The Interplay Between Coagulation and Inflammation Pathways in Covid-19-Associated Respiratory Failure: A Narrative Review. Pulm Ther 2020;6:215-31.

134. AIFA. Covid-19 - scheda informativa AIFA su eparine a basso peso molecolare. https:// www.aifa.gov.it/-/covid-19-scheda-informativa-aifa-su-eparine-a-basso-peso-molecolare. U1timo accesso: 1 febbraio 2021.
135. Goldenberg NA, Sochet A, Albisetti M, et al. Consensus-based clinical recommendations and research priorities for anticoagulant thromboprophylaxis in children hospitalized for Covid-19-related illness. J Thromb Haemost 2020;18:3099-105.

136. Chiotos K, Hayes M, Kimberlin DW, et al. Multicenter initial guidance on use of antivirals for children with Covid-19/SARS-CoV2. J Pediatric Infect Dis Soc 2020, Sept 12 [Epub ahead of print].

137. National Institute of Health. Coronavirus Disease 2019 (Covid-19) Treatment Guidelines. https://files.covid19treatmentguidelines.nih.go v/guidelines/covid19treatmentguide lines. pdf. Ultimo accesso: 18 novembre 2020.

138. Eghbali. Covid-19 in pediatric patients: a case series. J Cell Mol Anesth. 2020;5:3.

139. AIFA. Scheda su Remdesivir. https:// www.aifa.gov.it/documents/20142/1123276/r emdesivir update01_24.11.2020.pdf/f600b68a -7aea-6781-3dbf-934ddb269087c. Ultimo accesso: 1 febbraio 2021.

140. Remdesivir. Food and Drug Administration. 2020. Available at: https://www.accessdata.fda.gov/drugsatfda_docs/label/2020/2 14787Orig1s000lbl.pdf. Ultimo accesso il 18 novembre 2020.

141. Dong L, Hu S, Gao J. Discovering drugs to treat coronavirus disease 2019 (Covid-19). Drug Discov Ther 2020;14:58-60.

142. AIFA. Scheda su Lopinavir/ritonavir https://www.aifa.gov.it/documents/20142/1123 276/lopinavir_ritonavir_17.07.2020.pdf/ab9e0 7d8-585b-6eda-0007-a8f3d1e175c4. Ultimo accesso: 1 febbraio 2021.

143. AIFA. Scheda su Idrossiclorochina https://www.aifa.gov.it/documents/20142/1123 276/idrossiclorochina+update05_22.12.2020.p df/0d004070-a3e1-9aaa-7a0c-d30504fe19b8. Ultimo accesso: 1 febbraio 2021.

144. Harwood R, Allin B, Jones CE, et al.; PIMS-TS National Consensus Management Study Group. A national consensus management pathway for paediatric inflammatory multisystem syndrome temporally associated with Covid-19 (PIMS-TS): results of a national Delphi process. Lancet Child Adolesc Health 2021;5(2):133-41.

145. Henderson LA, Canna SW, Friedman KG, et al. American College of Rheumatology Clinical Guidance for Pediatric Patients with Multisystem Inflammatory Syndrome in Children (MIS-C) Associated with SARS-CoV-2 and Hyperinflammation in Covid-19. Version 2. Arthritis Rheumatol 2020, Dec 5 [Epub ahead of print]

146. Belhadjer Z, Méot M, Bajolle F, et al. Acute Heart Failure in Multisystem Inflammatory Syndrome in Children in the Context of Global SARS-CoV-2 Pandemic. Circulation 2020;142:429-36.

147. Dufort EM, Koumans EH, Chow EJ et al; New York State and Centers for Disease Control and Prevention Multisystem Inflammatory Syndrome in Children Investigation Team. Multisystem Inflammatory Syndrome in Children in New York State. N Engl J Med 2020;383:347-58.
148. Feldstein LR, Rose EB, Horwitz SM, et al. CDC Covid-19 Response Team. Multisystem Inflammatory Syndrome in U.S. Children and Adolescents. N Engl J Med 2020;383:334-46. 149. Chen S, Dong Y, Kiuchi MG, Wang J, et al. Coronary Artery Complication in Kawasaki Disease and the Importance of Early Intervention: A Systematic Review and Meta-analysis. JAMA Pediatr 2016;170:1156-63.

150. Ouldali N, Toubiana J, Antona D, et al. Association of Intravenous Immunoglobulins Plus Methylprednisolone vs Immunoglobulins Alone With Course of Fever in Multisystem Inflammatory Syndrome in Children. JAMA. Published online February 01, 2021. doi:10.1001/jama.2021.0694.

151. Capone CA, Subramony A, Sweberg T, et al. Characteristics, Cardiac Involvement, and Outcomes of Multisystem Inflammatory Syndrome of Childhood Associated with severe acute respiratory syndrome coronavirus 2 Infection. J Pediatr 2020;224:141-5.

152. Kone-Paut I, Cimaz R, Herberg J, et al. The use of interleukin 1 receptor antagonist (anakinra) in Kawasaki disease: A retrospective cases series. Autoimmun Rev 2018;17: 768-74.

153. Marchesi A, Tarissi de Jacobis I, et al. Kawasaki disease: guidelines of Italian Society of Pediatrics, part II - treatment of resistant forms and cardiovascular complications, follow-up, lifestyle and prevention of cardiovascular risks. Ital J Pediatr 2018;44:103.

154. Ferrara G, Giani T, Caparello MC, Farella C, Gamalero L, Cimaz R. Anakinra for Treatment- Resistant Kawasaki Disease: Evidence from a Literature Review. Paediatr Drugs 2020;22:645-52

155. de Graeff N, Groot N, Ozen S, et al. European consensus-based recommendations for the diagnosis and treatment of Kawasaki disease - the SHARE initiative. Rheumatology (Oxford) 2019;58:672-82.

156. Mahajan R, Lipton M, Broglie L, Jain NG, Uy NS. Eculizumab treatment for renal failure in a pediatric patient with Covid-19. J Nephrol 2020;33:1373-6.

157. Nakra NA, Blumberg DA, Herrera-Guerra A, Lakshminrusimha S. Multi-System Inflammatory Syndrome in Children (MIS-C) Following SARS-CoV-2 Infection: Review of Clinical Presentation, Hypothetical Pathogenesis, and Proposed Management. Children (Basel) 2020;7:69.

158. Chiang MH, Liu HE, Wang JL. Low-dose or no aspirin administration in acute-phase Kawasaki disease: a meta-analysis and systematic review. Arch Dis Child 2020, Nov 10 [Epub ahead of print].

159. The European Parliament. European Charter for children in Hospital A.1. Off J Eur Commun. 1986; 37-38 Doc A2-25/86 (No. C 148).

160. Kache S, Chisti MJ, Gumbo F, et al. Covid-19 PICU guidelines: for high- and limited resource settings. Pediatr Res 2020;88:705-16 161. Walker DM, Tolentino VR. Covid-19: The impact on pediatric emergency care. Pediatr Emerg Med Pract 2020;17:1-27. 
162. World Health Organization. Pocket Book for Hospital Care of Children: Guidelines for the Management of Common Illness with Limited Resources (World Health Organization, Geneva, 2013).

163. Khemani RG, Smith LS, Zimmerman JJ, Erickson S; Pediatric Acute Lung Injury Consensus Conference Group. Pediatric acute respiratory distress syndrome: definition, incidence, and epidemiology: proceedings from the Pediatric Acute Lung Injury Consensus Conference. Pediatr Crit Care Med 2015;16: S23-40.

164. Davis AL, Carcillo JA, Aneja RK, et al. The American College of Critical Care Medicine Clinical Practice Parameters for Hemodynamic Support of Pediatric and Neonatal Septic Shock: Executive Summary. Pediatr Crit Care Med 2017;18:884-90.

165. Workman JK, Ames SG, Reeder RW, et al. Treatment of Pediatric Septic Shock With the Surviving Sepsis Campaign Guidelines and PICU Patient Outcomes. Pediatr Crit Care Med 2016;17:e451-e458.

166. Health Advisory CDC. Multisystem Inflammatory Syndrome in Children (MIS-C) Associated with Coronavirus Disease 2019 (Covid-19). Atlanta, GA: CDC:2020. https:// emergency.cdc.gov/han/2020/han00432.asp? deliveryName=USCDC_511-DM28431 Ultimo accesso: 18 novembre 2020

167. Pediatric Acute Lung Injury Consensus Conference Group. Pediatric acute respiratory distress syndrome: consensus recommendations from the Pediartric Acute Lung Injury Consensus Conference. Pediatr Crit Care Med 2015;16:428-39.

168. Kneyber MCJ, Medina A, Alapont VM, et al. Practice recommendations for the management of children with suspected or proven $\mathrm{Co}_{-}$ vid-19 infections from the Paediatric Mechani- cal Ventilation Consensus Conference (PEMVECC) and the Section Respiratory Failure from the European Society for Paediatric and Neonatal Intensive Care (ESPNIC). https:// espniconline.org/Media/Files/2020- ESPNICPEMVECC-Covid-19-practice-recommendations. Ultimo accesso: 18 novembre 2020. 169. Hall D, Steel A, Heij R, Eley A, Young P. Videolaryngoscopy increases 'mouth-tomouth' distance compared with direct laryngoscopy. Anaesthesia 2020;75:822-3.

170. Matava CT, Kovatsis PG, Lee JK, et al. PeDI-Collaborative. Pediatric Airway Management in Covid-19 Patients: Consensus Guidelines From the Society for Pediatric Anesthesia's Pediatric Difficult Intubation Collaborative and the Canadian Pediatric Anesthesia Society. Anesth Analg 2020;131:61-73.

171. Rimensberger PC, Cheifetz IM, Pediatric Acute Lung Injury Consensus Conference Group. Ventilatory support in children with pediatric acute respiratory distress syndrome: proceedings from the Pediatric Acute Lung Injury Consensus Conference. Pediatr Crit Care Med 2015;16:S51-60.

172. Guérin C, Reignier J, Richard JC, et al. PROSEVA Study Group. Prone positioning in severe acute respiratory distress syndrome. N Engl J Med 2013;368:2159-68.

173. Weiss SL, Peters MJ, Alhazzani W, et al. Surviving Sepsis Campaign International Guidelines for the Management of Septic Shock and Sepsis-Associated Organ Dysfunction in Children. Pediatr Crit Care Med. 2020;21:e52e106.

174. Tamburlini M, Marchetti F, Bertino E, et al. Bambini e coronavirus: la doverosa ricerca di un equilibrio tra i presunti rischi e di documentati effetti collaterali. Medico e Bambino 2020;39:355-6.
175. Loades ME, Chatburn E, Higson-Sweeney N, et al. Rapid Systematic Review: The Impact of Social Isolation and Loneliness on the Mental Health of Children and Adolescents in the Context of Covid-19. J Am Acad Child Adolesc Psychiatry 2020;59:1218-39.e3.

176. Marchetti F. Gli effetti avversi del Covid19 in età pediatrica: la pandemia secondaria. Medico e Bambino pagine elettroniche 2020; 23:115-7.

177. Bignardi G, Dalmaijer ES, Anwyl-Irvine $\mathrm{AL}$, et al. Longitudinal increases in childhood depression symptoms during the Covid-19 lockdown. Arch Dis Child. 2020, Dec 9 [Epub ahead of print].

178. Wade M, Prime H, Browne DT. Why we need longitudinal mental health research with children and youth during (and after) the Covid19 pandemic. Psychiatry Res 2020;290: 113143. 179. Tamburlini G. L'impatto della pandemia Covid-19 sulla salute globale dei bambini. Medico e Bambino 2020;39:629-32.

180. Circolare Ministero Salute 13 gennaio 2021- Idoneità sportiva agonistica in atleti non professionisti CoVID-19 positivi guariti e in atleti con sintomi suggestivi per CoVID-19 in assenza di diagnosi da SARS-CoV-2 http:// www.sport.governo.it/media/2422/circolareidoneita-sportiva-np-covid-13-1-20.pdf.

181. Dean PN, Burns Jackson L, Paridon SN. Returning To Play After Coronavirus Infection: Pediatric Cardiologists' Perspectivehttps://www.acc.org/latest-in-cardiology/articles/2020/07/13/13/37/returning-to-play-after-coronavirus-infection. Ultimo accesso: 3 febbraio 2021.

182. Wade M, Prime H, Browne DT. Why we need longitudinal mental health research with children and youth during (and after) the Covid19 pandemic. Psychiatry Res 2020;290: 113143. 